\title{
Cortical neurotoxic astrocytes with early ALS pathology and miR-146a deficit replicate gliosis markers of symptomatic SOD1G93A mouse model
}

\section{Author's names}

Cátia Gomes ${ }^{1}$, Carolina Cunha ${ }^{1}$, Filipe Nascimento ${ }^{2}$, Joaquim A. Ribeiro ${ }^{2,3}$, Ana Rita Vaz ${ }^{1,4}$, Dora Brites ${ }^{1,4}$

\footnotetext{
Affiliations

${ }^{1}$ Research Institute for Medicines (iMed.ULisboa), Faculty of Pharmacy, Universidade de Lisboa, Lisbon, Portugal

${ }^{2}$ Unit of Neurosciences, the Institute for Molecular Medicine, Universidade de Lisboa, Lisbon, Portugal

${ }^{3}$ Institute of Pharmacology and Neurosciences, Faculty of Medicine, Universidade de Lisboa, Lisbon, Portugal

${ }^{4}$ Department of Biochemistry and Human Biology, Faculty of Pharmacy, Universidade de Lisboa, Lisbon, Portugal
}

\section{Corresponding author}

\section{Dora Brites}

Research Institute for Medicines (iMed.ULisboa)

Faculty of Pharmacy, Universidade de Lisboa

Av. Professor Gama Pinto, 1649-003 Lisbon, Portugal

Tel: +351217946450

Fax: +351217946491

E-mail address: dbrites@ff.ulisboa.pt 


\begin{abstract}
Amyotrophic lateral sclerosis (ALS) is a fatal neurodegenerative disease characterized by motor neuron (MN) loss. Recent evidences highlight astrocytes as important players in MN death, but the mechanismbased neurotoxicity is still unknown. It is also unclear whether activation of astrocytes in ALS occurs differently in the cerebral cortex and spinal cord. We investigated glial and neuronal alterations in the cortex of SOD1G93A (mSOD1) mice in pre-symptomatic and symptomatic stages. We also characterized astrocytes isolated from the cortex of 7-day-old mSOD1 mice for their aberrancy and MN-induced degenerative effects. In the early stage, we identified a reduction of cell proliferation, NF-kB expression, and of vimentin and micro(miR)-146a expression, suggesting a restrained cortical inflammatory status. However, increased NF-kB expression, cell proliferation, and gene expression of HMGB1, connexin-43, S100B were distinctive of the symptomatic stage, together with MN loss, downregulated unfold protein response, and decreased expression of synaptic proteins, miR-125b, miR-21, miR-146a, GFAP and glutamate transporters. Astrocytes cultured for 13 days-in-vitro showed comparable NF-kB expression and cell proliferation increase, as well as similar microRNA and gene expression profiles (decreased miR21, miR-146a, GLT-1 and GFAP, and upregulated HMGB1, S100B and connexin-43), thus sustaining astrocytes as the major contributors of cortical homeostasis deregulation in the symptomatic stage. These reactive astrocytes reduced neurite length and synaptophysin expression in NSC-34/hSODIWT MN-like cells, and induced mitochondria dysfunction, PSD-95 downregulation, metalloproteinase-9 activation and late apoptosis in NSC-34/hSOD1G93A cells. Data indicate that astrocytes in mSOD1 mice model acquire early phenotypic aberrancies and highlight downregulated miR-146a as a biomarker and drug target in
\end{abstract} ALS.

\title{
Keywords:
}

ALS astrocytes; Cerebral cortex; Inflammatory-associated microRNAs; Motor neurons; Pre-symptomatic and symptomatic stages; Transgenic $h S O D 1 G 93 A$ mice

\footnotetext{
Abbreviations:

ALS, amyotrophic lateral sclerosis; ATF4, activating transcription factor 4; ChAT, choline acetyltransferase; CNS, central nervous system; Cx43, connexin-43; DIV, days in vitro; ER, endoplasmic reticulum; fALS, familial ALS; GFAP, glial fibrillary acidic protein; GLAST, GLutamate ASpartate Transporter; GLT-1, glutamate transporter 1; GluT, glutamate transporters; HMGB1, high mobility group box 1; IRAK1, interleukin-1 receptor-associated kinase 1; LC3B, microtubule-associated protein light chain 3; MBP, myelin basic protein; miR, microRNA; MMP, matrix metalloproteinase; MN, motor neuron; mSOD1, mutant SOD1/SOD1G93A; NeuN, neuronal nuclei antigen; NF-kB, nuclear factor kappa B; NG2, neural/glial antigen 2; ON, overnight; PBS, phosphate buffer saline; eIF2a, eukaryotic initiation factor 2; PFA, paraformaldehyde; PN, postnatal; PSD-95, post-synaptic density protein 95; qPCR, quantitative real time-transcription polymerase chain reaction; RT, room temperature; S100B, S100 calcium binding protein B; sALS, sporadic ALS; SC, spinal cord; SOD1, superoxide dismutase 1; SYP, synaptophysin; TRAF6, tumor necrosis factor receptor-associated factor 6; WT, wild-type.
} 


\section{INTRODUCTION}

In amyotrophic lateral sclerosis (ALS), both upper and lower motor neurons (MN) die in the motor cortex, brainstem and spinal cord (SC) due to cell autonomous and non-cell autonomous mechanisms [1]. Although $90 \%$ of disease cases are sporadic (sALS), ALS is dominantly inherited in approximately $10 \%$ of patients (termed familial, fALS). Mutations in the antioxidant enzyme $\mathrm{Cu} / \mathrm{Zn}$ superoxide dismutase-1 (SOD1) gene was the first genetic link discovered in ALS [2] and account for 20-25\% of fALS cases [3]. In conformity, mice expressing the human SOD1 mutant (hSOD1G93A) are the most popular ALS standard model to investigate neuropathological features [4]. Although the specific mechanisms of toxicity remain unclear, several inflammatory pathways have been implicated in the spreading of the disease and MNs degeneration [5]. Most of these pathological mechanisms are due to cytoplasmic inclusions or aggregates in degenerating MNs, since they disrupt the normal protein homeostasis and promote cellular stress [6]. This abnormal protein aggregation seems to be a toxic feature in ALS, regardless of genotype, being observed in both fALS and sALS patients [7] and linked with decreased patient survival [8]. Moreover, wild-type (WT) MNs surrounded by mutant SOD1 (mSOD1)-expressing cells are severely damaged and undergo neurodegeneration [9]. Indeed, misfolded SOD1 inclusions were found in different glial cells of sALS and fALS patients [10] and selective ablation of mSOD1 expression in astrocytes slowed disease progression [11]. In addition, transplantation of SOD1G93A glial-restricted precursor cells into the cervical SC differentiated in neurotoxic astrocytes [12]. These findings clearly support the important role of astrocytes in ALS pathogenesis. Actually, astrocytes from both sALS and fALS patients, as well as from SOD1G93A rodent model, showed to be toxic to MNs $[13,14]$. Such cells, with a reactive profile, were identified in the primary motor cortex and gray matter from ALS disease cases $[15,16]$. These reactive astrocytes were characterized by their phenotypically aberrant profile, based on the high expression of S100 calcium binding protein B (S100B) and connexin 43 (Cx43) observed in the SC of SOD1G93A rats at the symptomatic stage, and were reported to induce the death of embryonic MNs [13]. Interestingly, Nagai et al. [17] have shown that mutant astrocytes from the SC of newborn pups, but not microglia, also cause specific MN toxicity, i.e. long before any visible reactive gliosis occurs. Although less numerous than those with SC astrocytes, previous studies examining reactive astrocytes in the cortical gray matter of non-motor and motor regions from cases of sALS and fALS revealed their widespread localization [16], and others pointed to their elevated expression on Cx43 [18]. Lately, we recognized immune-enriched microRNAs (miRNA), namely miRNA(miR)-124, miR-125b, miR-146a, miR-21 and miR-155 in the SC of symptomatic mSOD1 mice, together with upregulated nuclear factor kappa B (NF-kB)/NLRP3-inflammasome, HMGB1 and Cx43, and with downregulated glial fibrillary acidic protein (GFAP), glutamate aspartate transporter (GLAST) and glutamate transporter 1 (GLT-1) [19]. Differentially expressed miR-22, miR-155, miR-125b and miR-146b were found among the most highly modulated in microglia isolated from the brain cortex of SOD1G93A neonatal mice [20]. Moreover SC miR-155 was shown to be significantly upregulated in the SC of ALS patients, as well as in the mSOD1 mice microglia, and its inhibition to restore abnormal microglia and to prolong the survival of ALS mice [21,22]. Changes in miRNA expression were also linked to astrocyte reactivity [23] and increased astrocyte production of miR-146a to be a contributing factor in spinal muscular atrophy [24]. 
However, implication of astrocytic miRNA deregulation in ALS pathogenesis and neurodegeneration remain poorly investigated, and no studies have addressed inflammatory-associated miRNA profile in both cortical homogenates and isolated neonatal astrocytes from the mSOD1 mouse model.

Here, we examined the contribution of cortical astrocytes during disease progression, by assessing their pathophysiological reactive characteristics, including the inflammatory-associated miRNA profiling, by using tissue homogenates from the cortex of mSOD1 mice, in either pre-symptomatic (stage 1) or symptomatic stage (stage 2), as well as primary cultures of astrocytes isolated from the cortex of 7-dayold animals.

In the early stage, we found a decreased miR-146a expression and a restrained cortical inflammatory status. However, apparent markers of astrocyte aberrancy in stage 2, as reported by others [25], and downregulated miR-125b, miR-21 and miR-146a, are suggestive to contribute to the overwhelming hostile environment implicated in MN loss. Actually, most notorious symptomatic features were recapitulated in astrocytes isolated from the cortex of mSOD1 mice pups that were cultured for 13 daysin-vitro (DIV), namely the reduction of miR-146a expression. Such astrocytes produced harmful effects on either NSC-34/hSOD1WT or NSC-34/hSOD1G93A MN-cell line. Together, these data indicate that downregulation of miR-146a in cortical aberrant astrocytes may be a potential mechanism for astrocytemediated MN degeneration in ALS pathology.

\section{MATHERIAL AND METHODS}

\section{Ethics statement}

This study was performed in accordance with the European Community guidelines (Directives 86/609/EU and 2010/63/EU, Recommendation 2007/526/CE, European Convention for the Protection of Vertebrate Animals used for Experimental or Other Scientific Purposes ETS 123/Appendix A) and Portuguese Laws on Animal Care (Decreto-Lei 129/92, Portaria 1005/92, Portaria 466/95, Decreto-Lei 197/96, Portaria 1131/97). All the protocols used in this study were approved by the Portuguese National Authority (General Direction of Veterinary) and the Ethics Committee of the Instituto de Medicina Molecular (iMM) of the Faculty of Medicine, Universidade de Lisboa, Lisbon, Portugal.

\section{Animals}

Transgenic B6SJL-TgN (SOD1G93A)1Gur/J males (Jackson Laboratory, No. 002726) overexpressing the human SOD1 gene carrying a glycine to alanine point mutation at residue 93 (G93A) (mSOD1) [3] and WT B6SJLF1/J females were purchased from The Jackson Laboratory (Bar Harbor, ME, USA), and were bred at iMM rodent facilities, where a colony was established. Mice were maintained on the B6SJL background by breeding mSOD1 transgenic males with non-transgenic females in a rotational scheme. Males were crossed with non-transgenic females because transgenic females are infertile. Experiments were conducted in 7-day mice pups at postnatal day (PN) 7 and in two age groups: 4-6- and 12-14-weekold animals, corresponding to stage 1 (pre-symptomatic) and 2 (symptomatic), respectively. Such stages were previously established in the mSOD1mouse model based on the Rotarod test [26]. Both males and females were used since no gender influences over the intrinsic features of neuromuscular transmission 
were detected in this transgenic mice strain [27]. Transgenic mSOD1 mice were compared with agematched non-transgenic mice (WT) controls. The ear tissue extracted during the tagging procedure was used to genotype the progeny. For the 7-day-old mice, the tail was used to genotype the animals after sacrifice. Mice were housed at a maximum of 5 per cage, maintained under a $12 \mathrm{~h} \mathrm{light} / 12 \mathrm{~h}$ dark cycle, and received food and water ad libitum. Every effort was made to minimize the number and suffering of animals.

\section{Tissue slices and homogenates}

Animals were anesthetized with a lethal dose of sodium pentobarbital $(0.125 \mathrm{~g} / \mathrm{kg})$ and intracardially perfused. For quantitative real time-transcription polymerase chain reaction (qPCR) or Western blot, mice were perfused with $0.1 \mathrm{M}$ phosphate buffer (PBS) $\mathrm{pH} 7.4$ and cortical brain was dissected and rapidly frozen at $-80^{\circ} \mathrm{C}$. For tissue slices, mice were perfused with $4 \%$ paraformaldehyde (PFA, Sigma-Aldrich) in PBS; cortical brain was removed, post-fixed for $24 \mathrm{~h}$, and cryopreserved in 30\% sucrose. Sagittal 20$\mu \mathrm{m}$ thick sections were serially cut with the cryostat Leica CM1850 (UV Leica, Wetzlar, Germany).

\section{Primary culture of astrocytes}

Astrocytes were isolated from the cerebral cortices of 7-day-old WT and MSOD1 mice, as usual in our group [28], with minor modifications. Approximately, $2.5 \times 10^{4} \mathrm{cells} / \mathrm{cm}^{2}$ were plated on tissue culture plates in culture medium [DMEM (Biochrom AG, Berlin, Germany) supplemented with $11 \mathrm{mM}$ sodium bicarbonate (Merck, Darmstadt, Germany), 38.9 mM glucose (Merck), 1\% Antibiotic-Antimycotic solution (Sigma-Aldrich, St. Louis, MO, USA) and 10\% FBS (Biochrom AG)]. Cells were maintained at $37^{\circ} \mathrm{C}$ in a humidified atmosphere of $5 \% \mathrm{CO}_{2}$ until $13 \mathrm{DIV}$, with culture medium replacement at 7 and 10 DIV. Under these culture system conditions, microglia contamination was less than 5\%, which was revealed to not influence astrocyte inflammatory response [28], and that of oligodendrocytes and neurons only accounted for $4 \%$ and $1 \%$, respectively (Supplementary Table 1 ).

\section{Immunohistochemistry}

Cortical tissue cryosections (20- $\mu \mathrm{m}$ thickness) were defrosted at room temperature (RT) and fixed in cold acetone for $10 \mathrm{~min}$. Each section was blocked with $1 \%$ bovine serum albumin for $1 \mathrm{~h}$ at RT, and then incubated overnight $(\mathrm{ON})$ at $4^{\circ} \mathrm{C}$ with the primary antibodies (Supplementary Table 2). After washing, sections were covered with the secondary antibodies for $1 \mathrm{~h}$ at RT (Supplementary Table 2), and then incubated with DAPI for nuclei staining. Afterwards, brain sections were dehydrated in a series of ethanol solutions $(50 \%, 70 \%, 96 \%$ and 100\%), immersed in xylene, and mounted onto microscope slides using DPX. Finally, images of random microscopic fields from cortical brain were taken on a Leica DM6000 microscope (Leica Microsystems Heidelberg GmbH, Mannheim, Germany).

\section{Immunocytochemistry}

Immunofluorescent detection was performed as previously described by us [29]. After 13 DIV in culture, astrocytes growing in coverslips were fixed with 4\% PFA. Briefly, coverslips were incubated ON with primary antibodies (Supplementary Table 2). In the following day, after washes, coverslips were covered 
with species-specific fluorescent secondary antibodies (Supplementary Table 2). For nuclei staining, coverslips were incubated with Hoechst dye 33258 (Sigma-Aldrich) and then mounted onto uncoated slides using PBS-Glycerol (1:1). Finally, pairs of UV and fluorescence images of at least ten random microscopic fields were acquired per sample by using a fluorescence microscope (AxioSkope, Zeiss, Germany). For each protein marker we quantified the number of positive cells above a determined threshold of fluorescence per total number of cells. For Ki-67 staining, only cells with 2 or more nuclear punctuations were considered as positive.

\section{Western blot}

Western Blot analysis was performed as usual in our lab [28]. Briefly, total protein from primary astrocyte cultures were obtained by lysing cells in ice-cold Cell Lysis Buffer (Cell Signaling, Beverly, MA, USA), while cortex tissue was dissociated and protein extracted with RIPA (RadioImmunoprecipitation Assay) buffer. The protein extracts were separated on sodium dodecyl sulfatepolyacrylamide gel electrophoresis (SDS-PAGE) and transferred to a nitrocellulose membrane, which was blocked and incubated $\mathrm{ON}$ at $4^{\circ} \mathrm{C}$ with primary antibodies (Supplementary Table 2). In the following day, membranes were incubated with species-specific secondary antibodies conjugated to horseradish peroxidase (Supplementary Table 2). The chemiluminescent detection was performed after membrane incubation with LumiGLO ${ }^{\circledR}$ (Cell Signaling) or Western Bright ${ }^{\mathrm{TM}}$ Sirius (K-12043-D10, Advansta, Menlo Park, CA, USA). The relative intensities of the protein bands were analyzed using the ImageLab ${ }^{\mathrm{TM}}$ analysis software, after scanning with ChemiDocXRS, both from Bio-Rad Laboratories (Hercules, CA, USA). Results were normalized to the expression of $\beta$-actin and indicated as fold change $v s$. WT samples.

\section{Quantitative RT-PCR}

Total RNA was extracted from primary astrocyte cultures and cerebral cortical sections using TRIzol ${ }^{\circledR}$ Reagent (LifeTechnologies) and following manufacturer's instructions. Total RNA was quantified using Nanodrop ND-100 Spectrophotometer (NanoDrop Technologies, Wilmington, DE, USA), and conversion to complementary DNA (cDNA) was performed with a cDNA Synthesis Kit (Thermo Scientific). Quantitative RT-PCR (qRT-PCR) was accomplished on a 7300 Real Time PCR System (Applied Biosystems) using SYBR Green qPCR Master Mix (Thermo Scientific). qRT-PCR was performed under optimized conditions: $94^{\circ} \mathrm{C}$ for $3 \mathrm{~min}$ followed by 40 cycles at $94^{\circ} \mathrm{C}$ for $0.15 \mathrm{~min}, 62^{\circ} \mathrm{C}$ for $0.2 \mathrm{~min}$ and $72^{\circ} \mathrm{C}$ for $0.15 \mathrm{~min}$. In order to verify the specificity of the amplification, a melt-curve analysis was performed immediately after the amplification protocol. Non-specific products of PCR were not found in any case. $\beta$-actin was used as an endogenous control to normalize the expression level.

Expression of miRNAs was also assessed by qRT-PCR. After RNA quantification, cDNA conversion for miRNA quantification was performed with the universal cDNA Synthesis Kit (Exiqon), as described [19], using $5 \mathrm{ng}$ total RNA. qRT-PCR was performed using the above indicated 7300 Real Time PCR System and 96-well plates. For miRNA quantification the miRCURY LNATM Universal RT miRNA PCR system (Exiqon, Vedbaek Denmark) was used in combination with pre-designed primers (Exiqon). The reaction conditions consisted of polymerase activation/denaturation and well-factor determination at $95^{\circ} \mathrm{C}$ for $10 \mathrm{~min}$, followed by 50 amplification cycles at $95^{\circ} \mathrm{C}$ for $10 \mathrm{~s}$ and $60^{\circ} \mathrm{C}$ for $1 \mathrm{~min}$ (ramp-rate of $1.6^{\circ} / \mathrm{s}$ ). 
SNORD110 (reference gene) was used as an endogenous control to normalize the expression level. Primer sequences are listed in the Supplementary Table 3. Relative mRNA/miRNA concentrations were calculated using the $\Delta \Delta \mathrm{C}_{\mathrm{T}}$ equation, and results were expressed as fold change $v s$. WT samples.

\section{Extracellular HMGB1}

The release of HMGB1 was determined in the conditioned media of astrocyte cultures using the HMGB1 Detection Kit (catalog\#6010, Gentaur Molecular Products, Kampenhout, Belgium), following manufacturer's instructions. Briefly, the capture antibody was placed in a 96-well plate and incubated ON at $4^{\circ} \mathrm{C}$. In the following day, after adding the detection antibody, each collected cell medium or the HMGB 1 standard was applied. The plate was incubated for $1 \mathrm{~h}$ at $37^{\circ} \mathrm{C}$ and then for approximately $20 \mathrm{~h}$ at $4^{\circ} \mathrm{C}$. Afterwards, streptavidin peroxidase was added in each well and maintained at RT for $30 \mathrm{~min}$. Then, the TMB was placed in each well and the plate incubated at RT for $30 \mathrm{~min}$. Finally, the stop solution ( $2 \mathrm{~N}$ sulfuric acid) was added into each well. Optical density values were red within $30 \mathrm{~min}$ in a microplate absorbance spectrophotometer (PR 2100, BioRad Laboratories, Inc.) at $450 \mathrm{~nm}$ (sample) and $630 \mathrm{~nm}$ (reference filter). Results were expressed as fold change $v s$. WT samples.

\section{Cell death determination}

Phycoerythrin-conjugated Annexin V (Annexin V-PE) and 7-amino-actinomycin D (7-AAD; Guava Nexin ${ }^{\circledR}$ Reagent, \#4500-0450, Millipore) were used to determine the percentage of viable (Annexin V-PE and 7-AAD negative), early-apoptotic (Annexin V-PE positive and 7-AAD negative), late stages of apoptotic cells (Annexin V-PE and 7-AAD positive) and necrotic cells/debris (Annexin V-PE negative and 7-AAD positive) by flow cytometry. After incubation, cells were trypsinized and added to the cells in supernatants following manufacturer's instructions. Then they were stained with Annexin V-PE and 7AAD and analyzed on a Guava easyCyte 5HT flow cytometer (Guava Nexin ${ }^{\circledR}$ Software module, Millipore). Results were expressed as percentage of positive cell population.

\section{Assessment of astrocyte-induced toxicity on motor neurons}

Co-cultures of 13 DIV astrocytes and NSC-34 MN-like cells were used to determine the neurotoxic properties of astrocytes. As so, NSC-34 cells expressing either the WT human SOD1 (WT MNs) or the G93A mutation (mSOD1 MNs) were included [29]. Cells were maintained for $48 \mathrm{~h}$ in proliferation media [DMEM high glucose, w/o pyruvate, supplemented with $10 \%$ of FBS and $1 \%$ of penicillin/streptomycin, and geneticin sulfate $(\mathrm{G} 418,0.5 \mathrm{mg} / \mathrm{ml})$ for selection], followed by additional $48 \mathrm{~h}$ in differentiation media [DMEM-F12 plus FBS (1\%), nonessential amino acids (1\%) and penicillin/streptomycin (1\%)], as previously indicated by us [29]. Cortical WT or mSOD1 13 DIV astrocytes were seeded onto paraffin dots-containing coverslips [30], which were placed on top of a layer of WT or mSOD1 NSC-34 MNs, in the proportion of 1:1. Cells were co-cultured for $48 \mathrm{~h}$, and then harvested separately for neurotoxicassociated determinations, such as cell death (as described before), expression of synaptophysin (SYP) and post-synaptic density protein 95 (PSD-95) by Western blot (as described above) and expressed as fold change vs. WT Ast/MN co-culture, as well as for the assessment of mitochondria viability, metalloproteinase-9 (MMP-9) and neurite extension, as indicated below. 


\section{Assessment of mitochondria membrane potential}

In order to analyze mitochondria membrane potential, NSC-34 MNs were incubated for $30 \mathrm{~min}$ at $37^{\circ} \mathrm{C}$ with $500 \mathrm{nM}$ of MitoTracker ${ }^{\mathrm{TM}}$ Red CMXRos solution as usual in our lab [29], and then fixed with 4\% PFA. Cell nuclei were stained with Hoechst 33258 dye. Images were acquired as described above for immunocytochemistry. Fluorescence intensity was quantified by ImageJ software and normalized to the total number of cells.

\section{Determination of MMP-9 activation}

MMP-9 extracellular levels were determined by SDS-PAGE zymography in $0.1 \%$ gelatin-10\% acrylamide gels, under non-reducing conditions [29]. Briefly, following electrophoresis, MMP species were renatured with $2.5 \%$ Triton-X-100 (in $50 \mathrm{mM}$ Tris $\mathrm{pH} 7.4 ; 5 \mathrm{mM} \mathrm{CaCl} 2 ; 1 \mu \mathrm{M} \mathrm{ZnCl2}$ ). To induce gelatin lysis, gels were incubated in the same buffer without Triton $\mathrm{X}-100 \mathrm{ON}$ at $37^{\circ} \mathrm{C}$. Afterwards, gels were stained with $0.5 \%$ Coomassie Brilliant Blue R-250 and distained in 30\% ethanol/10\% acetic $\mathrm{acid} / \mathrm{H}_{2} \mathrm{O}$. Gelatin activity was detected as a white band on a blue background and measured by ImageLab software. Results were expressed as fold change vs. WT Ast/MN co-culture.

\section{Neurite extension determination}

Neurite extension was assessed by the immunofluorescence detection of the cytoskeletal protein $\beta$ IIItubulin using a mouse anti- $\beta$ III-tubulin as the primary antibody (information in Supplementary Table 2) and according with the immunocytochemistry assay described before. The neurite extension in individual neurons was measured using ImageJ software, as previously described [29].

\section{Statistical analysis}

Results of at least four different experiments were expressed as mean values \pm SEM. Results of astrocyte cultures were represented as fold $v s$. WT samples. Results of cortical tissue samples from mSOD1 mice at stages 1 or 2 were represented as fold $v s$. age-matched WT mice. Differences between groups were determined by the two-tailed unpaired Student's $t$-test with Welch's correction, depending on whether variances were equal or different, respectively. Exceptionally, it was used one-way ANOVA followed by Bonferroni (selected pairs of columns) post-hoc test in Figure 1b and one-way ANOVA followed by Tukey's multiple comparison post-hoc test in Figure 6b. Differences between the effect of WT and mSOD1 astrocytes in WT MNs was obtained by two-tailed unpaired Student's $t$-test with Welch's correction when applicable. The same methodology was applied for mSOD1 MNs. Statistical analysis was performed using GraphPad PRISM 5.0 (GraphPad Software, San Diego, CA, USA). $p<0.05$ was considered as statistically significant.

\section{RESULTS}

Cortical accumulation of SOD1 protein precedes downregulation of unfolded protein response in the symptomatic stage 
Abnormal accumulation of SOD1 is a hallmark of ALS and one of the reasons for endoplasmic reticulum (ER) stress. Enhanced eIF2 $\alpha$ phosphorylation in the mSOD1 mice ameliorates the disease course, because eukaryotic initiation factor 2 (eIF2 $\alpha$ ) phosphorylation and the subsequent activation of transcription factor 4 (ATF4) prevent accumulation of unfolded and misfolded SOD1 protein [31]. However, the inhibition of eIF2 $\alpha$ phosphorylation by using the GSK2606414 compound, that knockdowns PERK, was shown to retain climbing ability in flies expressing TDP-43 and to mitigate TDP-43 toxicity in rat primary neurons [32]. Different results may derive from diverse ALS stages being used in such studies. Therefore, we decided to evaluate SOD1 accumulation, as well as phosphorylated-eIF2 $\alpha$ and ATF4, in the mSOD1 mice at stage 1 (pre-symptomatic: 4-6 weeks old) and stage 2 (symptomatic: 12-14 weeks old), using agematched WT animals as controls. As depicted in Figure 1a, the fluorescence intensity for SOD1 in the brain cortex was higher in the mSOD1 mice than in the WT animals and occurred in both stages, although at elevated levels in the stage 2. Such finding was corroborated by the western blot analysis of cortical homogenates, using an anti-SOD1 antibody that recognized either human or mouse SOD1 $(p<0.05$, Fig. 1b). WT mice only exhibited one band corresponding to the native mouse SOD1, as previously described [19]. Now considering the phosphorylated eIF2 $\alpha$ and the transcription factor ATF4, we observed that both were reduced, but only in mSOD1 mice at stage 2 ( $p<0.01$, Fig. 1c and d, respectively), thus enhancing the risk of SOD1 accumulation. Evidences have shown that eIF2 $\alpha$-ATF4 pathway is essential for autophagy gene transcription program in response to stresses [33]. However, probably due to the deregulated eIF2 $\alpha$-ATF4 pathway observed, we were unable to identify any alteration in the expression of microtubule-associated protein light chain 3 (LC3B) and Beclin-1, used as autophagy-related markers, in either disease stages (Supplementary Figure 1).

\section{Neurodegeneration, reduced levels of synaptic proteins and decreased miR-125b characterize the symptomatic stage in the cerebral cortex}

Given that increased mSOD1 accumulation causes early defects in MN outgrowth and axonal branching [34], and that reduced ATF4 leads to abnormal synaptic plasticity [35] while enhancing mSOD1 aggregation and decreasing motor performance in ALS mice [36], we analyzed neuronal fate, as well as pre- and post-synaptic protein expression. To determine neuronal viability in the cortex of mSOD1 mice in both stages 1 and 2, we assessed the expression of neuronal nuclei antigen (NeuN) protein, which is localized in nuclei and in perinuclear cytoplasm of most neurons of the central nervous system (CNS), and choline acetyltransferase (ChAT), a generic MN marker [37]. The decreased expression of both NeuN and ChAT proteins at stage 2 ( $p<0.05$, Fig. 2a and b, respectively), indicates a marked loss of neurons, mainly of MNs ( 40\%). Then, when we assessed the expression of the pre-synaptic (synaptophysin, SYP) and post-synaptic (post-synaptic density protein 95, PSD-95) proteins, we observed that both were also significantly reduced at stage 2 ( $p<0.05$, Fig. $2 c$ and d, respectively). Interestingly, PSD-95 levels were found increased in the early stage 1 ( $p<0.05$, Fig. 2d). Such upregulation, never documented in ALS, may result from a compensatory mechanism similar to that previously noticed in Alzheimer's disease, where it was related with a primary stage and preceding the diminution of the postsynaptic protein [38]. Moreover, since miR-124 was shown to regulate synaptic structure and function [39] and miR-125b to be associated with neurite outgrowth [40], we evaluated their representation in 
cortical tissue. Though, no changes were observed for miR-124 expression (Fig. 2e), a significant reduction was obtained for miR-125b ( $p<0.01$, Fig. 2f) at stage 2 that may be associated to the decreased expression of neuronal markers also observed in the same disease stage.

\section{Cortical miR-146a downregulation is an early event that precedes miR-21 decrease and elevation of NF-kB, HMGB1 and Ki-67 gene expression at the symptomatic stage}

Since NF-kB signaling is critical in neuroinflammation and was shown to cause MN death in ALS models [41], we determined whether NF-kB changes were associated to neurodegeneration and inflammatory microenvironment in the cortex of the ALS mouse, either prior or after disease onset. We observed that total NF-kB protein expression was decreased in the stage 1 ( $p<0.05$, Fig. 3a), a condition suggested to compromise its neuroprotective role [42], though it can also contribute to counteract the neuroinflammatory initiation status. In opposite, NF-kB upregulation in stage $2(p<0.05)$ was shown to contribute to neural cell death after injury [43]. However, when phospho-NF-kB was determined, no significant alterations were observed (Fig. 3b), although an increasing trend with disease progression was noticed, corroborating previous findings in SC samples [41]. As HMGB1 is currently accepted as a regulator of NF-kB signaling pathway [44], we decided to assess whether changes in the gene and protein expression of such alarmin could be related with the opposed NF-kB expression profiles in stages 1 and 2. HMGB1 mRNA levels were only increased at stage $2(p<0.001$, Fig. 3c), and no changes were observed relatively to its protein expression (Fig. 3d), what may indicate either a failure in protein synthesis or increased degradation. Actually, decreased HMGB1 immunoreactivity was previously detected in the ventral horn MNs of the mSOD1 mice, and suggested to derive from cell loss or extracellular release [45]. NF-kB was also indicated to be an important promoter of cell proliferation [46], reason why we further evaluated the expression of Ki-67. Interestingly, we observed the same profile as the one obtained for NF$\mathrm{kB}$, with a downregulation at stage $1(p<0.05)$, followed by an upregulation at stage $2(p<0.01)$, as depicted in Figure 3e. To additionally note that when we compared differences from the stage 1 to the stage 2, either in WT or in mSOD1 animals, the first group evidenced Ki-67 decrease $(p<0.05)$, while the later one showed an increase $(p<0.05)$ (data not shown), thus reinforcing the occurrence of increased cell proliferation with disease progression. Then, and because miRNAs were shown to play a critical role in fine tuning neuroinflammation and indicated to be differentially expressed in ALS [47], we assessed if miR-21 and miR-146a were deregulated in the cortical tissue of mSOD1 mice, therefore accounting for the inflammatory milieu. Actually, their reduced expression was shown to cause elevated NF-kB levels and increase of pro-inflammatory factors [48], reason why they are considered as negative regulators of inflammation. As expected, we found a reduced expression of both miRNAs at stage $2(p<0.05$, Fig. $3 \mathrm{f}$ and g). However, the decrease of miR-146a was also identified as a very first feature, since it was already significant at stage $1(p<0.05)$. Such finding highlights miR-146a as an early biomarker of the disease and a promising target for drug development toward prevention of stage 2 inflammatory-related factors, since it was considered a suppressor of cellular immune response [49].

\section{Oligodendrocytes and microglia are slightly modified by the disease in the cerebral cortex}


Several studies have described the role of glial cells in MN degeneration and loss, defining ALS as a noncell autonomous disease [1]. Moreover, analysis of samples from sALS and fALS patients, revealed misfolded SOD1 inclusions in different glial cells [10]. Such inclusions were related with astrocyte reactivity, microglia activation and $\mathrm{MN}$ degeneration [50]. Lately, oligodendrocytes were also shown to have a SOD1-dependent pathogenic phenotype in ALS [51]. We first looked for the expression of early oligodendrocyte progenitor marker NG2 and no changes in mRNA levels or protein expression were detected (Supplementary Figure $2 \mathrm{~b}$ and a, respectively). However, expression of myelin basic protein (MBP), was found reduced in the stage $1(p<0.05$ ), but not in stage 2 (Supplementary Figure 2c), despite early studies referring its decrease in the symptomatic stage [52]. Differences may arise from regional and phenotypic heterogeneity, as previously described for cortical microglia not showing the atypical phenotype of spinal cells [53]. Indeed, we were unable to observe changes in Cd11b cortical microglia, in either stages of the disease in the mSOD1 mice (Supplementary Figure 2d). To corroborate the absence of microglia activation in the brain cortex of mSOD1 mice, we did not observe any alteration in miR-155 expression (Supplementary Figure 2e), indicated as highly expressed by activated microglia [54]. To even note that when the comparison was made between the stage 1 and stage 2 only in the mSOD1 mice, expression of miR-155 was found decreased in the later period ( $p<0.05$, data not shown), which suggests a decrease in the expression of miR-155 along disease progression in the cerebral cortex.

\section{Cortical upregulation of $S 100 B / C x 43$ and downregulated glutamate transporters/GFAP expression in the symptomatic stage suggest astrocyte reactivity and deregulation}

Alterations in the astrocytic population have extensively been evidenced in ALS [55] due, in part, to SOD1 inclusions. In particular, Diaz-Amarilla et al. [13] described a specific phenotype of reactive and neurotoxic astrocytes in the SC of mSOD1 rats. However the identification of these reactive cells in the cortex has been poorly studied and the mechanisms by which they become deregulated are still unknown. Since the results suggest that oligodendrocytes or microglia are not evidently contributing to the neurodegeneration observed in the symptomatic stage 2, we decided to analyze the expression of astrocytes markers. As depicted in Figure 4a, S100B immunofluorescence intensity was higher in the cortical tissue of mSOD1 mice at the stage 2, where it was accompanied by elevated S100B mRNA levels ( $p<0.01$, Fig. 4 b). When we compared differences from the stage 1 to the stage 2 only in the mSOD1 animals, a 3-fold elevation ( $p<0.05$ ) was obtained in the later period (data not shown), thus showing that rising levels of S100B correlate with disease progression. In addition, considering that elevated S100B and $\mathrm{Cx} 43$ are markers of astrocyte reactivity $[13,56]$ and that their abnormal expression was attributed to mSOD1 astrocytes-mediated neurotoxicity [57,18], we further assessed Cx43 gene expression. As we anticipated, a marked elevation was found in stage 2 ( $p<0.01$, Fig. $4 \mathrm{c})$.

Taking into account that dysfunction of astroglial glutamate transporters (GluT), i.e. GLT-1 and GLAST, was observed in the SC of mSOD1 rodents [13], we investigated their expression in the cortical samples. As depicted in Figure 5a, GLT-1 immunofluorescence was decreased in tissue extracts of mSOD1 mice at stage 2, and both GLT-1 and GLAST protein levels were found decreased in the same period $(p<0.05$, Fig. $5 \mathrm{~b}$ and c, respectively). Because loss of GLT-1 was suggested to be associated with overexpressed GFAP [58], and previous studies revealed that vimentin was expressed in normal levels, while GFAP was 
reduced by $60 \%$ in ALS aberrant astrocytes [13], we next evaluated the expression of such intermediate filaments in the cerebral cortex homogenates. Curiously, we identified a reduced cortical vimentin expression in the early stage 1 of the mSOD1 mice ( $p<0.001$, Fig. $5 \mathrm{~d}$ ), which disappeared with disease progression. Conversely, a decreased GFAP immunofluorescence and protein expression $(p<0.05$, Fig. $5 \mathrm{e}$ and $\mathrm{g}$, respectively) were observed in the cortical tissue of mSOD1 mice, but only in stage 2. However, reduction of GFAP mRNA was only identified in stage 1 ( $p<0.05$, Fig. 5f). These findings may contribute to deficient astrocyte motility [59] and to accelerate ALS progression [60,13].

Because our data highlighted the potential role of astrocytes for the reactive profile we found in the cortical brain of mSOD1 mice during the symptomatic stage, we next evaluated astrocyte phenotypic profile and aberrancy when isolated from the same animals and region at 7-day-old.

\section{Astrocytes isolated from the cortex of mSOD1 mice pups exhibit an abnormal and reactive phenotype with gain of gliosis markers and reduced expression of GFAP and GLT-1}

Astrocytes isolated from cortices at 0-2 day-old mSOD1 mice have previously exhibited enhanced reactivity [61], though those of mSOD1 rodents isolated from the SC of 1day-old pups revealed to express lower levels of S100B and Cx43, and more GLT-1, than those obtained from the adult SC of symptomatic animals [13]. Nevertheless, it was lately shown that astrocytes from the cerebral cortices of 7-8 day-old mice P301S tau transgenic mice possess an abnormal phenotype that is retained in adulthood, in contrast with those from 1 to 2 day-old mice, indicating that the astrocytic reaction can take some days to develop [62]. Therefore, in order to analyze the profile of mSOD1 astrocytes and how they associate with the features observed in the cortex of adult mice in the symptomatic stage 2 , we decided to isolate the cells from the cortex of 7-day-old mSOD1 and WT mice. Cells were used for experiments after 13 DIV.

Concerning SOD1 levels, almost $100 \%$ of astrocytes derived from mSOD1 mice presented SOD1 inclusions, as compared with only $10 \%$ found in WT astrocytes ( $p<0.001$, Fig. 6a). Assessment of SOD1 protein expression similarly revealed a 6 -fold increase relatively to that of WT animals $(p<0.001$, Fig. 6b). Since such elevated SOD1 expression is known to account for astrocyte deregulation and neurotoxicity [11], we next analyzed markers related with the characteristic astrocyte aberrancy in the SC of mSOD1 rodents [13], most of them also found in the cortical tissue during the symptomatic stage, as demonstrated in the previous sections of this paper. As expected, cultured astrocytes showed increased S100B immunofluorescence $(p<0.01$, Supplementary Fig. 3) and gene expression $(p<0.05$, Fig. 6c), Cx43 mRNA levels ( $p<0.01$, Fig. 6d), vimentin expression ( $p<0.05$, Fig. 6j and Supplementary Fig. 3), as well as enhanced Ki-67 immunofluorescent positive cells $(p<0.01$, Supplementary Fig. 3 ) and gene expression $(p<0.05$, Fig. 6 e), relatively to the WT astrocytes, thus corroborating the typical reactive profile noticed in the cortical tissue collected from the mSOD1 mice at the symptomatic stage 2. Also in accordance, astrocytes presented not only lower GFAP gene expression and protein levels $(p<0.05$, Fig. 6f and g, and Supplementary Fig. 3), but also decreased GLT-1 ( $p<0.05$, Fig. 6h and Supplementary Fig. 3 ) and GLAST ( $p<0.05$, Fig. 6i, and Supplementary Fig. 3) expression levels, than respective controls. Notably, loss of GFAP expression in astrocytes was not caused by cell death, since no difference was obtained for early apoptosis between cells isolated from either WT, or mSOD1 mice, and viable cells 
accounted for more than $90 \%$ of total number of astrocytes (Supplementary Table 4). Collectively, we may then consider that astrocytes isolated from the cortex of mSOD1 mice pups show SOD1 accumulation and present a set of specific markers (GFAP ${ }^{\text {low }} / \mathrm{GLT}-1^{\text {low }} / \mathrm{GLAST}^{\text {low }} / \mathrm{S} 100 \mathrm{~B}^{\text {high }} / \mathrm{Cx} 43^{\text {high }} / \mathrm{Ki}$ $67^{\text {high} / v i m e n t i n ~}{ }^{\text {high }}$ ) that characterize such cells as having an atypical reactive phenotype, which is early acquired (Fig. 6 and Supplementary Fig. 3).

Astrocytes isolated from the cortex of mSOD1 mice pups show upregulation of the NFkB/HMGB1 signaling pathway and deficits in miR-21 and miR-146a expression levels

Previous studies have shown that lipopolysaccharide or interleukin(IL)-1 $\beta$-stimulated astrocytes upregulate HMGB1 protein expression and its release [63,64]. On the other way, astrocytes also acquire a specific reactive phenotype, related with a pro-inflammatory-like response, when stimulated with HMGB1 [65], which was demonstrated to activate NF-kB signaling [44]. To clarify whether the increased NF-kB expression and HMGB1 mRNA levels found in the cortical tissue of mSOD1 mice at stage 2 could be attributed to astrocytes, we decided to explore this issue. Cultured astrocytes revealed increased expression ( $p<0.05$, Fig. 7a) and phosphorylation of NF-kB ( $p<0.05$, Fig. 7b), as well as NF-kB translocation to the nucleus (Fig. 7c). Moreover, we also observed increased HMGB1 gene expression $(p$ $<0.05$, Fig. 7d) and its release to cell supernatants ( $p<0.01$, Fig.7f), probably justifying why no changes were observed for HMGB1 intracellular protein levels (Fig. 7e). Such results further validate the reactive profile of these cells.

Upregulated expression of miR-146a was found in areas of prominent gliosis and in astrocytes-enriched human cell cultures stimulated with IL-1 $\beta$ upon human, and its effects associated to a regulation of astrocyte-mediated inflammatory response [49]. Additionally, Bhalala et al. [66] described that miR-21 overexpression in astrocytes attenuated the hypertrophic profile, while miR-21 inhibition led to increased hypertrophic phenotype, suggesting increased reactivity linked with low miR-21 levels. Importantly, both miR-21 and miR-146a inhibit expression of genes that repress cell proliferation and promote apoptosis and their balance plays a critical role in neuronal survival, at least after SC injury [67]. Therefore, it was quite surprising to find out that these miRNAs were subexpressed in the cortical tissue of the mSOD1 mice at the symptomatic stage, so we were interested in determining the contribution of astrocytes to such dysregulation. Actually, both miR-21 ( $p<0.05$, Fig. 7g) and miR-146a ( $p<0.01$, Fig. $7 \mathrm{~h})$ were deficiently expressed in the astrocytes with an abnormal and reactive phenotype. Results demonstrate that mSOD1derived astrocytes acquire early deficits that are retained during ALS progression.

In order to validate that miR-146a decrease was related with an elevation of its target genes, interleukin-1 receptor-associated kinase 1 (IRAK1) and TNF receptor-associated factor 6 (TRAF6), we next assessed their mRNA expression. They are commonly identified as negatively regulated by miR-146a and implicated in NF-kB activation [48]. As expected, both IRAK1 and TRAF6 showed to be upregulated in mSOD1 astrocytes ( $p<0.05$, Fig. $7 \mathrm{i}$ and $\mathrm{j}$, respectively) supporting that miR-146a downregulation impacts on TLR/NF-kB signaling, thus contrubuting to neuroinflammation.

Reactive and deregulated astrocytes isolated from mSOD1 mice pups exert diverse toxic effects on WT and mSOD1 MNs that involve synaptic failure 
Several studies have demonstrated that human/mouse astrocytes derived from sALS or fALS models cause MN degeneration and loss $[12,14,11,68]$. Recently, it was demonstrated that reactive astrocytes, independent of genetic mutations, reduce MN survival, then indicating that they may have a major role in both sALS and fALS [69]. However, when astrocytes derived from sALS patients and healthy induced pluripotent stem cells were transplanted into the cervical SC of adult severe combined immunodeficiency mice, Authors found that only the first cause ALS-like degeneration in both MNs and non-MNs [68]. Here we have shown that ALS astrocytes, besides showing reactive markers, have a specific phenotype with low levels of GFAP, GluT and, overall, a dysfunctional miR-146a and miR-21 profile. To add that the large majority of these studies have only analyzed the effect of ALS astrocytes on healthy MNs, disregarding the effect they may produce on the already dysfunctional ALS MNs.

Hence, we established co-cultures of astrocytes isolated from the cortex of mSOD1 mice pups showing early ALS pathology, with NSC-34 MN-like cells, either expressing WT human SOD1 (WT MNs), or containing the G93A mutation (mSOD1 MNs) [29]. By assessing neurite extension after $48 \mathrm{~h}$ of coculturing, it was notorious that mSOD1 astrocytes caused shorter neurite length ( $p<0.05$, Fig. 8a), and SYP subexpression ( $p<0.05$, Fig. 8b) in WT MNs, but not in mSOD1 MNs, when compared to matched WT astrocytes. This not without precedent, since ALS astrocytes were reported to promote neurite shortening [55,14], and synaptic failure [70]. Mitochondria dynamic abnormalities and activation of MMP-9 are well known features of ALS MNs [29], but ever highlighted to be potentially aggravated by ALS astrocytes, as we evidence in the present study. Indeed, mSOD1 astrocytes led to decreased mitochondrial membrane potential in mSOD1 MNs ( $p<0.01$, Fig. 8d), together with an increased release of MMP-9 $(p<0.05$, Fig. 8 c). Both results indicate that mutated MNs are highly susceptible to such toxic effects by mSOD1 astrocytes. To reinforce their vulnerability, we also found a marked reduction on the post-synaptic protein PSD-95 ( $p<0.01$, Fig. 8e) and a manifest increase of cells showing late apoptosis $(p<0.01$, Fig. 8f). Data show that astrocytes revealing ALS pathology compromise the functionality of both mSOD1 and WT MNs, and that the produced harmful effects depend on MN selective susceptibility. In any case, astrocytes should be envisaged as targets of combined therapeutic strategies aimed at different aspects of their dysfunctional phenotype in ALS.

\section{Discussion}

Contribution of glial cells to neurotoxic events in ALS has been extensively reported. Regardless of being a first or a secondary event, dysfunctional astrocytes have been identified as key players in ALS and MN degeneration [13,55]. Brain cortex has been poorly studied in ALS when compared with the extensive works on SC pathology. However, increasing evidences highlight the relevance of cortical region, based on delayed disease onset and extended survival in mSOD1 rat model by the suppression of mutant SOD1 in such region [71]. Moreover, once such Authors suggest dyingforward progression in ALS with a particular impact on the brain cortex our study on cortical glial cells reactivity and neurotoxic potential was designed to identify new targets and signaling pathways in neurodegeneration. In this work we highlight alterations that characterize a more reactive, dysfunctional and neurotoxic population of cortical 
astrocytes, which are early found in cells isolated from the 7-day-old mSOD1 mice and cultured for 13 DIV. Actually, we identified a specific astrocytic phenotype characterized by concomitant increased expression of NF-kB, SOD1, Cx43, S100B, HMGB1 and Ki-67, and decreased GFAP, GLT-1, miR-146a and miR-21 levels. These parameters were similarly denoted in the cortical tissue samples of symptomatic mice (12-14 weeks-old animals).

Though SOD1 accumulation was observed in both mSOD1 mice disease stages, it increased from stage 1 to stage 2, suggesting that it contributes to disease progression. Accumulation of mSOD1 and misfolded SOD1 was previously shown to occur in both sALS and fALS [7], and to be age-dependent and tissue specific [72]. Abnormal accumulation of SOD1 is a hallmark of ALS and one of the reasons for ER stress. Some works refer the decrease in eIF $2 \alpha$ phosphorylation and repression of the transcription factor ATF4 as favoring the accumulation of the unfolded and misfolded SOD1 protein, since enhanced eIF2 $\alpha$ phosphorylation in the mSOD1 mice was found to ameliorate the course of the disease [31,73]. However, other studies found increased levels and benefits after therapeutic modulation [32,74]. Different results may derive from diverse ALS stages being used for such studies. Our findings indicate that reduced eIF$2 \alpha$ phosphorylation and consequent decrease in ATF4 expression translates in SOD1 aggregation in the cortical tissue of the mSOD1 mice at stage 2. Thus, our data is in line with preceding studies demonstrating that ATF4 deficiency promotes mSOD1 aggregation at the disease end-stage [36] and that decreased eIF $2 \alpha$ phosphorylation interferes with the suppression of protein synthesis and triggers mSOD1 aggregation [31].

SOD1 mutations and protein misfolding have been associated to inflammatory and neurotoxic pathways in ALS [5]. Neuronal NeuN, and more specifically ChAT-positive MNs, were found reduced in the symptomatic stage of mSOD1 mice, as mentioned before [75]. When we investigated whether neurodegeneration was affecting synaptic integrity and cortical connectivity, we observed that both SYP and PSD-95 were decreased in stage 2. Decreased levels of PSD-95, but not SYP, were identified in the postmortem human cortical tissue of ALS patients [76], though other previous studies in the SC of ALS patients also revealed a decrease of SYP with aging [77]. Elevated expression of PSD-95 at stage 1 may represent a compensation mechanism, once the magnitude of the excitatory effects on neuromuscular transmission [38], caused by A2A receptor, was found enhanced at this stage, but decreased in stage 2 [27]. Isolated cortical oligodendrocytes from mSOD1 mice were also identified as contributing to axonal dysfunction in ALS [51]. Nevertheless, we only noticed a decreased MBP expression at stage 1 and no alterations for NG2 expression in either stage.

Deregulation of several inflamma-miRs has been associated with ALS onset and progression [78,47]. Specifically, in what concerns miR-124 and miR-125b, both recognized as being enriched in the nervous system tissue and neural cells [79], we found a decreased expression of miR-125b and a slight reduction of miR-124. Interestingly, miR-124 was shown to regulate synaptic structure and function [39], while miR-125b revealed to promote neurite outgrowth [40]. Moreover, since upregulation of miR-125b was demonstrated to favor regeneration after SC injury [80], we may assume that the decreased miR-125b we found in cortical brain tissue further compromises MN survival. This finding together with the signal transmission failure deriving from the impaired synaptic protein expression, will account to aggravate neurodegeneration. In what concerns miR-155, whose upregulation is associated with microglia activation 
in the SC, and pointed as a promising therapeutic target in ALS [22,19], we were unable to identify any alteration in the cortical tissue samples. Actually, no microglia activation based on Cd11b expression was even detected in cortical homogenates suggesting the presence of regional variants and microglia phenotypic variability in ALS, which may have implications when deciding therapeutic strategies. Among other miRNAs suspected to contribute to ALS pathology is the altered expression of miR-146a and miR-21in the SC and brainstem of ALS transgenic mice [81]. Similarly to these authors we also found reduced miR-146a levels in the cortical homogenates, a feature not observed in the SC from the same mSOD1 mice model, in either stage 1 or stage 2 [19]. Again, our results contrast with other studies showing an elevated miR-146a expression in patient SC samples [82], suggesting the existence of phenotypic changes in ALS patients and rodent models, while supporting the new label of precision medicine. Our data further revealed miR-146a as a powerful ALS biomarker due to its early appearance before symptom onset and maintenance along disease progression, at least in the brain cortex. Notably, reduced miR-21 was lately identified in the CSF of ALS patients, and also suggested as a potential biomarker of the disease [83]. Supporting the role of miR-146a and miR-21 as negative regulators of the TLR/NF-kB inflammatory pathway [48], increased expression in NF-kB levels was a feature of symptomatic stage 2, though elevated HMGB1, as we observed, was also described to promote its activation [44] and previously reported in reactive SC glia from ALS patients [84]. It is worthwhile to note that a restrained cortical activation/inflammatory status may coexist with miR-146a deficit in stage 1 , based on the decreased GFAP and Ki-67 mRNA, as well as on the reduced NF-kB expression, which was similarly observed in the SC of the mSOD1 mice at the pre-symptomatic stage [19], and hypothesized to have a role in preventing disease onset. In fact, some in vitro and in vivo works demonstrated that the inhibition of NF-kB in astrocytes does not increase motor function or survival in mSOD1 mice [85,41]. However, the in vivo NF-kB inhibition was performed by over-expressing the dominant negative form of $\mathrm{IkB} \alpha$ selectively in GFAP-positive astrocytes (GFAP-IkBaAA). Indeed, similarly with what was reported to SC astrocytes [13], we are describing a population of neurotoxic cortical astrocytes that show decreased GFAP levels. Therefore, the selectively inhibition of NF-kB in astrocytes taking into account the GFAP expression could not be targeting the harmful astrocytes, or at least, their inhibition could not be enough to ameliorate disease progression. Moreover, the study of Frakes and colleagues [41] shows that the inhibition of NF-kB in SC astrocytes, in vitro, does not prevent MNs loss, though authors did not validate this finding in cortical SOD1G93A derived astrocytes.

Phenotypically aberrant astrocytes were shown to promote $\mathrm{MN}$ damage and intracellular protein aggregation in ALS models [13,69]. Such astrocytes revealed to be hypertrophic and to contain decreased GFAP and high levels of S100B and Cx43 [13,19]. Because most of the studies have been carried out on $\mathrm{SC}$, we here explored markers associated to reactive astrocytes on the cortical brain homogenates from mSOD1 mice at stage 1 and stage 2 . In line with those studies we found the same kind of alterations only when samples were collected during the symptomatic stage, excepting GFAP mRNA, whose reduced expression was present already in stage 1. Decrease of GFAP was shown to accelerate ALS progression [60] and its coexistence with vimentin reduction to impact on neurite network and synaptic loss [86]. Consistent reduced GFAP levels associated to reactive and neurotoxic astrocytes have been highlighted in samples from mSOD1 models or astrocytes transfected with the mutated protein $[19,13,87]$. Another 
well-known feature in ALS is the impairment in GluT considered to contribute to the excitotoxic path of MN degeneration $[13,88]$. Decreased expression of GLT-1 and GLAST was also manifest in the cortical tissue samples from our mSOD1 mice model, after disease appearance. Collectively, these results highlight that the atypical biomarkers characteristic of the aberrant reactive astrocytes are predominate and are the main players in the pathophysiological processes occurring in the brain cortex of mSOD1 ALS mice at the symptomatic stage.

To test whether astrocytes are intrinsically deficient and acquire an early reactive phenotype when isolated during the first postnatal week of life, as recently demonstrated for the AD P301S tau mice model [62], we decided to explore all the above markers of astrocytic activation in cells isolated from 7 day-old mSOD1 mice pups and cultured for 13 DIV. Our primary cultures of astrocytes revealed a pathological phenotype characterized by reactive/proliferative properties (S100B ${ }^{\text {high }}, \mathrm{Cx} 43^{\text {high }}, \mathrm{Ki}-67^{\text {high }}$, vimentin high $)$ and dysfunctional features (GFAP ${ }^{\text {low }}$, GLT-1 ${ }^{\text {low }}$, GLAST ${ }^{\text {low }}$ ). Such findings contrast with published data on neonatal astrocytes isolated from mSOD1 rat newborns [13] indicating that 7 day-old pups, but not 1-2 day-old mice, develop pathological changes as indicated by Sidoryk-Wegrzynowicz et al. [62]. This concept is further supported by the increased expression and activation of NF-kB, elevated HMGB1 mRNA and release of HMGB1 protein, together with decreased miR-146a and miR-21 expression levels in mSOD1 astrocytes, thus recapitulating major findings observed in cortical tissue from symptomatic mice, including the SOD1 accumulation. Data on ALS cortical astrocytes additionally sustained downregulated miR-146a as a pivotal player in the early pathological events, inasmuch as decreased miR146a expression has been reported to mediate inflammatory response [49], and to promote astrocyte proliferation [89], as we observed. Accordingly, our preliminary data on the benefits of miR-146a upregulation indicated a reduction in aberrancy ALS astrocyte biomarkers (data not shown). Indeed, miR-146a was shown to act as a negative-feedback regulator of the inflammatory pathway, and to directly target IRAK1 and TRAF6, both downstream components of the TLR cascade and mediators of inflammation via NF-kB activation [90,48]. Other studies revealed that miR-146a by downregulating IRAK1 and TRAF6 in macrophages reduced the production of pro-inflammatory cytokines [91]. We then hypothesize that miR-146a downregulation is directly associated or contributes to astrocyte-inflammatory potential and extracellular neurotoxic milieu. To highlight, however, that such decrease seems to be specific of the cerebral cortex since it was not found in the SC [19]. Therefore, miR-146a may be considered a potential therapeutic target to revert the reactive phenotype of astrocytes in ALS.

Considering that ALS cortical astrocytes early express a set of biomarkers characteristic of cell reactivity, which may compromise neuronal survival, neurite extension and synaptic signaling, we evaluated these parameters in NSC-34 WT and mutated SOD1 MNs after exposure to such diseased astrocytes isolated, as previously, from the 7 day-old mice pups and cultured for 13 DIV. Our data on the effects of reactive astrocytes toward WT MNs revealed that they cause a reduction in neurite length, as previously documented for directly converted sALS and fALS astrocytes over mouse embryonic stem cellsdifferentiated MNs [14], indicating that an impairment of synaptic transmission is produced. Contribution of astrocytes in compromising mediating synaptic plasticity was shown to occur in the presence of inflammatory cytokines [92]. Interestingly, here we show that reactive/deregulated astrocytes directly affect SYP and consequently synaptic signaling between adjacent neurons. To mention that such features 
were not observed in mSOD1 MNs, indicating that diseased astrocytes differently distress WT and ALS MNs. In conformity, other alterations, such as increased MMP-9 activity and late apoptosis, together with mitochondria dysfunction and reduced PSD-95 expression, were exclusively observed in mSOD1 MNs after co-culturing with reactive/deregulated ALS primary astrocytes. These findings are important in ALS if we consider that MMP-9 is recognized to be a determinant of selective neurodegeneration [93], decreased levels of PSD-95 to determine a dysfunctional post-synaptic terminal [76], and damaged mitochondria to be a sign of neuronal vulnerability [29], here translated in late apoptosis. Different signatures of neurotoxicity in response to ALS astrocytes are therefore manifest in either WT, or mSOD1 MNs, suggesting unequally vulnerability of healthy and diseased neurons to astrocyte-induced neurotoxicity.

Overall, our study provides advanced evidence that astrocytes lose neurosupportive functions in brain cortex of mSOD1 mouse model and gain neurotoxic properties, probably accounting for the neuronal loss and synaptic dysfunction, together with predominant reactive markers associated to astrocyte phenotypic aberrancies that we show here to characterize the symptomatic stage of mSOD1 rodent models. Data also reveal that the low expression of miR-125b, miR-21 and miR-146a may account for neurite degeneration, synaptic impairment, worse neurological outcome and defective regulation of activation and inflammatory status after disease onset. In addition, this study stands out for the first time that downregulated miR-146a is one of the earliest predictive biomarkers of the disease, with specificity for the brain cortex, since it was not found in the SC [19]. Moreover, our findings indicating that no microglia activation, or miR-155 upregulation, or even any significant alteration on oligodendrocytes are present in the mSOD1 mice cortical region, emphasize astrocytes as key players and raise the question on whether microglia in SC and brain cortex may be differently associated to disease progression in ALS. Actually, cortical astrocytes show to acquire early deficits and to induce neurodegeneration while revealing all the reactive markers identified in the symptomatic stage. Most notoriously, besides expressing low miR-146a and miR-21 levels, astrocytes showed to release HMGB1 into the extracellular milieu, which is a well-known mediator of neuroinflammation and neurodegeneration [94]. Harmful effects were consequently produced on either NSC-34/hSOD1WT or NSC-34/hSODIG93A MNs, though by different pathways, highlighting that astrocyte phenotypic aberrancies include a broad-spectrum of action. Future work should address astrocytes in the cerebral cortex for neuroprotective and neurorestorative treatment strategies, and consider miR-146a deficiency as a pivotal biomarker and new therapeutic target in ALS.

\section{Acknowledgments}

This work was supported by the Research Grant of the Santa Casa Scientific Research Program on ALS, by Santa Casa da Misericórdia de Lisboa (SCML), Portugal, Project Ref. ELA-2015-002 (to DB), by the project PTDC/SAU-FAR/118787/2010 (to DB) and, in part, by iMed.ULisboa (UID/DTP/04138/2013) from Fundação para a Ciência e a Tecnologia (FCT). CG and CC are recipients of PhD fellowships from FCT (SFRH/BD/102718/2014 and SFRH/BD/91316/2012, respectively) and ARV of a Postdoctoral grant (SFRH/BPD/76590/2011). The funding organization had no role in study design, data collection and 
analysis, decision to publish, or preparation of the manuscript. We thank Dr ${ }^{\mathrm{a}}$ Andreia Barateiro and $\mathrm{Dr}^{\mathrm{a}}$ Ana Domingos for acquisition of brain section images in Leica DM6000 inverted Confocal Microscope at Instituto Gulbenkian de Ciência (Oeiras, Portugal).

\section{Conflict of Interest Statement}

The authors declare that the research was conducted in the absence of any potential conflict of interest.

\section{Figure legends}

Fig. 1 SOD1 accumulation is associated with decreased eIF-2 $\alpha$ phosphorylation and ATF4 expression in the cerebral cortex of mSOD1 mice at symptomatic stage. Cortical tissue samples were obtained from SOD1G93A (mSOD1) and wild type (WT) mice at 4-6 weeks-old (pre-symptomatic, stage 1) and 12-14 weeks-old (symptomatic, stage 2). (a) Representative fluorescence images of superoxide dismutase-1 (SOD1) staining (green) by immunohistochemistry. (b) Western blot analysis of SOD1 expression (mouse, light gray bars; human, dark gray); the anti-SOD1 antibody recognize both human and mouse SOD1. Western blot analysis showing the expression levels of (c) phospho-eukaryotic initiation factor 2 (p-eIF-2 $\alpha$ ) and (d) activating transcription factor 4 (ATF4). Representative results from one blot are shown. $\beta$-actin was used as a loading control. Results are represented as fold vs. age-matched WT animals, except in $\mathbf{b}$ where the results are represent as fold $v s$. mouse SOD1 in stage 1. Data represent mean values $\pm \mathrm{SEM}$ from at least five independent experiments. $* * p<0.01 v s$. WT samples, two-tailed unpaired Student's $t$-test with Welch's correction when required. Exceptionally for SOD1 analysis (in b), ${ }^{\#} p<0.05$ vs. human SOD1 in stage 1, one-way ANOVA followed by Bonferroni (selected pairs of columns) post-hoc test. Scale bar represents $40 \mu \mathrm{m}$.

Fig. 2 Cortical tissue samples from symptomatic mSOD1 mice show neuronal degeneration/loss, as well as reduced expression of synaptic proteins and miR-125b. Cortical tissue samples were obtained from SOD1G93A (mSOD1) and wild type (WT) mice at 4-6 weeks-old (pre-symptomatic, stage 1) and 12-14 weeks-old (symptomatic, stage 2). Western blot analysis showing the expression levels of (a) neuronal specific nuclear protein $(\mathrm{NeuN})$, (b) choline acetyltransferase (ChAT), (c) pre-synaptic synaptophysin (SYP) and (d) post-synaptic density protein 95 (PSD-95). Representative results from one blot are shown. $\beta$-actin was used as a loading control. qPCR gene expression analysis of (e) microRNA(miR)-124 and (f) miR-125b. Results are represented as folds vs. age-matched WT mice. Data represent mean values \pm SEM from at least five independent experiments. ${ }^{*} p<0.05$ and $* * p<0.01$ vs. WT samples, two-tailed unpaired Student's $t$-test with Welch's correction when was required.

Fig. 3 Inflammatory status in cortical tissue samples from mSOD1 mice at symptomatic stage is preceded by depressed stress markers and miR-146a expression. Cortical tissue samples were obtained from SOD1G93A (mSOD1) and wild type (WT) mice at 4-6 weeks-old (pre-symptomatic, stage 1) and 12-14 weeks-old (symptomatic, stage 2). Western blot analysis showing the expression levels of (a) nuclear factor kappa B (NF-kB), (b) phosphor-NF-kB and (d) high mobility group box 1 (HMGB1). Representative results from one blot are shown. $\beta$-actin was used as a loading control. qPCR gene 
expression analysis of (c) HMGB1, (e) proliferation marker (Ki-67), (f) microRNA(miR)-21 and (g) miR146a. Results are represented as folds vs. age-matched WT mice. Data represent mean values \pm SEM from at least five independent experiments. ${ }^{*} p<0.05$, $* * p<0.01$ and $* * * p<0.001$ vs. WT samples, twotailed unpaired Student's $t$-test, with Welch's correction when was required.

Fig. 4 Elevated expression of $\mathrm{S} 100 \mathrm{~B}$ and $\mathrm{Cx} 43$ astrocytic markers are observed in cortical tissue samples from mSOD1 mice at symptomatic stage. Cortical tissue samples were obtained from SOD1G93A (mSOD1) and wild type (WT) mice at 4-6 weeks old (pre-symptomatic, stage 1) and 12-14 weeks old (symptomatic, stage 2). Representative fluorescence images of (a) S100 calcium-binding protein B (S100B) staining (red) by immunohistochemistry. qPCR gene expression analysis of (b) S100B and (c) connexin-43 (Cx43). Results are represented as folds vs. age-matched WT mice. Data represents mean values \pm SEM from at least five independent experiments. ${ }^{*} p<0.01 v s$. WT samples, two-tailed unpaired Student's $t$-test. Scale bar represents $40 \mu \mathrm{m}$.

Fig. 5 Decreased GFAP mRNA and vimentin expression precede the occurrence of downregulated glutamate transporters and astrocyte GFAP expression levels in the mSOD1 mice at symptomatic stage. Cortical tissue samples were obtained from SOD1G93A (mSOD1) and wild type (WT) mice at 4-6 weeks old (pre-symptomatic, stage 1) and 12-14 weeks old (symptomatic, stage 2). Representative fluorescence images of (a) Glutamate Transporter 1 (GLT-1) and (e) glial fibrillary acidic protein (GFAP) staining by immunohistochemistry. Western blot analysis showing the expression levels of (b) GLT-1, (c) GLutamate ASpartate Transporter (GLAST), (d) vimentin and (g) GFAP. Representative results from one blot are shown. $\beta$-actin was used as a loading control. qPCR gene expression analysis of (f) GFAP levels. Results are represented as folds $v s$. age-matched WT mice. Data represent mean values \pm SEM from at least five independent experiments. ${ }^{*} p<0.05$ and $* * * p<0.001 v s$. respective WT samples, two-tailed unpaired Student's $t$-test with Welch's correction when was required. Scale bar represents $40 \mu \mathrm{m}$.

Fig. 6 Cortical astrocytes isolated from the mSOD1 mice show an atypical reactive phenotype characterized by SOD1 accumulation, increased gliosis markers and reduced GFAP and GLT-1 expression. Astrocytes were isolated from the cortex of SOD1G93A (mSOD1) and wild type (WT) mice pups at 7 day-old, and cultured for 13 days in vitro. (a) Representative fluorescence images of superoxide dismutase-1 (SOD1) staining (green) by immunocytochemistry. (b) Western blot analysis of SOD1 expression (mouse, light gray bars; human, dark gray); the anti-SOD1 antibody recognize both human and mouse SOD1. qPCR gene expression analysis of (c) S100 calcium-binding protein B (S100B), (d) connexin-43 (Cx43), (e) Ki-67 and (f) glial fibrillary acidic protein (GFAP). Western blot analysis showing the expression levels of (g) GFAP, (h) GLT-1, (i) GLutamate ASpartate Transporter (GLAST) and (j) vimentin. Representative results from one blot are shown. $\beta$-actin was used as a loading control. Results are represented as folds vs. WT mice. Data represent mean values \pm SEM from at least five independent experiments. $* p<0.05$ and $* * p<0.01 v s$. WT samples, two-tailed unpaired Student's $t$-test with Welch's correction when was required. Exceptionally for SOD1 analysis (in b), ***p < $0.001 v s$. 
mouse SOD1 in WT samples, one-way ANOVA followed by Tukey's multiple comparison post-hoc test. Scale bar represents $40 \mu \mathrm{m}$.

Fig. 7 Cortical astrocytes isolated from the mSOD1 mice show upregulated NF-kB/HMGB1 signaling, and decreased miR-21 and miR-146a levels. Astrocytes were isolated from the cortex of SOD1G93A (mSOD1) and wild type (WT) mice pups at 7 day-old, and cultured for 13 days in vitro. Western blot analysis showing the expression levels of (a) nuclear factor kappa B (NF-kB), (b) phosphoNF-kB and (e) high mobility group box 1 (HMGB1). Representative results from one blot are shown. $\beta$-actin was used as a loading control. (c) Representative fluorescence images of NF-kB staining (green) by immunocytochemistry, to assess translocation to the nucleus. qPCR gene expression analysis of (d) HMGB1, (g) microRNA(miR)-21, (h) miR-146a, (i) interleukin-1 receptor-associated kinase 1 (IRAK1) and (j) tumor necrosis factor receptor-associated factor 6 (TRAF6). (f) ELISA analysis of HMGB1 released by astrocytes into the extracellular media. Results are represented as folds vs. WT mice. Data represent mean values \pm SEM from at least five independent experiments. $* p<0.05$ and $* * p<0.01$ $v s$. WT samples, two-tailed unpaired Student's $t$-test with Welch's correction when was required. Scale bar represents $40 \mu \mathrm{m}$.

Fig. 8 Atypical reactive astrocytes isolated from the cerebral cortex of mSOD1 mice show neurotoxic effects on WT and mSOD1 MNs. Astrocytes were isolated from the cortex of SOD1G93A (mSOD1) and wild type (WT) mice pups at 7 day-old, and cultured for 13 days in vitro. WT and mSOD1 astrocytes (Ast) were co-cultured with WT and mSOD1 NSC-34 MNs for 48 hours in vitro. Representative fluorescence images of (a) neurite extension ( $\beta$ III-Tubulin) and (d) MitoTracker ${ }^{\mathrm{TM}}$ Red CMXRos staining by immunocytochemistry. Western blot analysis showing the expression levels of (b) synaptophysin (SYP) and (e) post-synaptic protein density 95 (PSD-95) proteins after exposure to astrocytes. Representative results from one blot in which samples ran in the same gel with same conditions are shown. $\beta$-actin was used as a loading control. (c) Gelatin zymography analysis of matrix metalloproteinase-9 (MMP-9) activity in the cell co-culture supernatants. (f) Percentage of positive cells for Annexin(+) and 7-AAD(+) (indicator of late-apoptotic/necrotic cells) in WT and mSOD1 MNs assessed by Guava Nexin ${ }^{\circledR}$ Reagent. Results are represented as folds vs. respective MNs (WT or mSOD1) co-cultured with WT Ast (in b, $\mathbf{c}$ and $\mathbf{e}$ ). Data represent mean values \pm SEM from at least five independent experiments. ${ }^{*} p<0.05$ and ${ }^{*} p p<0.01$ using two-tailed unpaired Student's $t$-test Welch's correction when was required. Scale bar represents $40 \mu \mathrm{m}$.

Supplementary Figure 1 Unchanged LC3B and Beclin-1 protein expression in the cerebral cortex of mSOD1 mice indicate that the autophagy process is not affected by the disease. Cortical tissue samples were obtained from SOD1G93A (mSOD1) and wild type (WT) mice at 4-6 weeks-old (presymptomatic, stage 1) and 12-14 weeks-old (symptomatic, stage 2). Western blot analysis showing the expression levels of (a) microtubule-associated protein 1 light chain 3B (LC3B) and (b) Beclin-1. Representative results from one blot are shown. $\beta$-actin was used as a loading control. Results are 
represented as fold $v s$. WT animals in the same disease stage. Data represent mean values \pm SEM from at least five independent experiments.

Supplementary Figure 2 Expression levels of proteins associated with oligodendrocyte progenitors and activated microglia in the cerebral cortex of mSOD1 mice are not affected by the disease, though the population of mature myelinating oligodendrocytes is reduced before disease onset. . Cortical tissue samples were obtained from SOD1G93A (mSOD1) and wild type (WT) mice at 4-6 weeks-old (pre-symptomatic, stage 1) and 12-14 weeks-old (symptomatic, stage 2). Western blot analysis showing the expression levels of (a) NG2 and (c) myelin basic protein (MBP). Representative results from one blot are shown. $\beta$-actin was used as a loading control. qPCR gene expression analysis of (b) NG2 , (d) CD11b mRNA and (e) microRNA(miR)-155 levels. Results are represented as folds vs. agematched WT mice. Data represent mean values \pm SEM from at least five independent experiments. * $p<$ 0.05 vs. WT samples, two-tailed unpaired Student's $t$-test with Welch's correction when was required.

Supplementary Figure 3 Astrocytes isolated from the cerebral cortex of mSOD1 mice show a predominance of cells globally presenting an atypical reactive phenotype. Astrocytes were isolated from the cortex of SOD1G93A (mSOD1) and wild type (WT) mice pups at 7 day-old, and cultured for 13 days in vitro. Representative fluorescence images of S100 calcium-binding protein B (S100B), the proliferation marker Ki-67, fibrillary acidic protein (GFAP), glutamate transporter 1 (GLT-1), GLutamate ASpartate Transporter (GLAST) and, vimentin and Respective quantification based on the number of positive cells above a fluorescent signal threshold settled for each marker. For Ki-67 staining, only cells with 2 or more nuclear punctuations were considered as positive. Results are represented as folds vs. WT samples. Data represent mean values \pm SEM from at least five independent experiments. $* p<0.05$, **p $<0.01$ and $* * * p<0.001$ vs. WT samples, two-tailed unpaired Student's $t$-test with Welch's correction when was required. Scale bar represents $40 \mu \mathrm{m}$.

\section{References}

1. Bento-Abreu A, Van Damme P, Van Den Bosch L, Robberecht W (2010) The neurobiology of amyotrophic lateral sclerosis. Eur J Neurosci 31 (12):2247-2265. doi:10.1111/j.14609568.2010.07260.x

EJN7260 [pii]

2. Rosen DR (1993) Mutations in Cu/Zn superoxide dismutase gene are associated with familial amyotrophic lateral sclerosis. Nature 364 (6435):362. doi:10.1038/364362c0

3. Gurney ME, Pu H, Chiu AY, Dal Canto MC, Polchow CY, Alexander DD, Caliendo J, Hentati A, Kwon YW, Deng HX, et al. (1994) Motor neuron degeneration in mice that express a human $\mathrm{Cu}, \mathrm{Zn}$ superoxide dismutase mutation. Science 264 (5166):1772-1775

4. Pfohl SR, Halicek MT, Mitchell CS (2015) Characterization of the Contribution of Genetic Background and Gender to Disease Progression in the SOD1 G93A Mouse Model of Amyotrophic Lateral Sclerosis: A Meta-Analysis. J Neuromuscul Dis 2 (2):137-150. doi:10.3233/JND-140068 
5. McCombe PA, Henderson RD (2011) The Role of immune and inflammatory mechanisms in ALS. Curr Mol Med 11 (3):246-254

6. Saxena S, Caroni P (2011) Selective neuronal vulnerability in neurodegenerative diseases: from stressor thresholds to degeneration. Neuron 71 (1):35-48. doi:10.1016/j.neuron.2011.06.031

7. Gruzman A, Wood WL, Alpert E, Prasad MD, Miller RG, Rothstein JD, Bowser R, Hamilton R, Wood TD, Cleveland DW, Lingappa VR, Liu J (2007) Common molecular signature in SOD1 for both sporadic and familial amyotrophic lateral sclerosis. Proc Natl Acad Sci U S A 104 (30):12524-12529. doi:10.1073/pnas.0705044104

8. Wang Q, Johnson JL, Agar NY, Agar JN (2008) Protein aggregation and protein instability govern familial amyotrophic lateral sclerosis patient survival. PLoS Biol 6 (7):e170. doi:10.1371/journal.pbio.0060170

9. Clement AM, Nguyen MD, Roberts EA, Garcia ML, Boillee S, Rule M, McMahon AP, Doucette W, Siwek D, Ferrante RJ, Brown RH, Jr., Julien JP, Goldstein LS, Cleveland DW (2003) Wild-type nonneuronal cells extend survival of SOD1 mutant motor neurons in ALS mice. Science 302 (5642):113-117. doi:10.1126/science.1086071

10. Forsberg K, Andersen PM, Marklund SL, Brannstrom T (2011) Glial nuclear aggregates of superoxide dismutase- 1 are regularly present in patients with amyotrophic lateral sclerosis. Acta Neuropathol 121 (5):623-634. doi:10.1007/s00401-011-0805-3

11. Yamanaka K, Chun SJ, Boillee S, Fujimori-Tonou N, Yamashita H, Gutmann DH, Takahashi R, Misawa $\mathrm{H}$, Cleveland DW (2008) Astrocytes as determinants of disease progression in inherited amyotrophic lateral sclerosis. Nat Neurosci 11 (3):251-253. doi:10.1038/nn2047

nn2047 [pii]

12. Papadeas ST, Kraig SE, O'Banion C, Lepore AC, Maragakis NJ (2011) Astrocytes carrying the superoxide dismutase 1 (SOD1G93A) mutation induce wild-type motor neuron degeneration in vivo. Proc Natl Acad Sci U S A 108 (43):17803-17808. doi:10.1073/pnas.1103141108

1103141108 [pii]

13. Diaz-Amarilla $P$, Olivera-Bravo $S$, Trias E, Cragnolini A, Martinez-Palma L, Cassina $P$, Beckman J, Barbeito L (2011) Phenotypically aberrant astrocytes that promote motoneuron damage in a model of inherited amyotrophic lateral sclerosis. Proc Natl Acad Sci U S A 108 (44):18126-18131. doi:10.1073/pnas.1110689108

14. Meyer K, Ferraiuolo L, Miranda CJ, Likhite S, McElroy S, Renusch S, Ditsworth D, LagierTourenne C, Smith RA, Ravits J, Burghes AH, Shaw PJ, Cleveland DW, Kolb SJ, Kaspar BK (2014) Direct conversion of patient fibroblasts demonstrates non-cell autonomous toxicity of astrocytes to motor neurons in familial and sporadic ALS. Proc Natl Acad Sci U S A 111 (2):829832. doi:10.1073/pnas.1314085111

1314085111 [pii]

15. Kamo H, Haebara H, Akiguchi I, Kameyama M, Kimura H, McGeer PL (1987) A distinctive distribution of reactive astroglia in the precentral cortex in amyotrophic lateral sclerosis. Acta Neuropathol 74 (1):33-38

16. Nagy D, Kato T, Kushner PD (1994) Reactive astrocytes are widespread in the cortical gray matter of amyotrophic lateral sclerosis. J Neurosci Res 38 (3):336-347. doi:10.1002/jnr.490380312

17. Nagai M, Re DB, Nagata T, Chalazonitis A, Jessell TM, Wichterle H, Przedborski S (2007) Astrocytes expressing ALS-linked mutated SOD1 release factors selectively toxic to motor neurons. Nat Neurosci 10 (5):615-622. doi:nn1876 [pii]

$10.1038 / \mathrm{nn} 1876$ 
18. Almad AA, Doreswamy A, Gross SK, Richard JP, Huo Y, Haughey N, Maragakis NJ (2016) Connexin 43 in astrocytes contributes to motor neuron toxicity in amyotrophic lateral sclerosis. Glia 64 (7):1154-1169. doi:10.1002/glia.22989

19. Cunha C, Santos C, Gomes C, Fernandes A, Correia AM, Sebastiao AM, Vaz AR, Brites D (2017) Downregulated Glia Interplay and Increased miRNA-155 as Promising Markers to Track ALS at an Early Stage. Mol Neurobiol:doi: 10.1007/s12035-12017-10631-12032. doi:10.1007/s12035-017-0631-2

10.1007/s12035-017-0631-2 [pii]

20. Parisi C, Arisi I, D'Ambrosi N, Storti AE, Brandi R, D'Onofrio M, Volonte C (2013) Dysregulated microRNAs in amyotrophic lateral sclerosis microglia modulate genes linked to neuroinflammation. Cell Death Dis 4:e959. doi:10.1038/cddis.2013.491

cddis2013491 [pii]

21. Koval ED, Shaner C, Zhang P, du Maine X, Fischer K, Tay J, Chau BN, Wu GF, Miller TM (2013) Method for widespread microRNA-155 inhibition prolongs survival in ALS-model mice. Hum Mol Genet 22 (20):4127-4135. doi:10.1093/hmg/ddt261

ddt261 [pii]

22. Butovsky O, Jedrychowski MP, Cialic R, Krasemann S, Murugaiyan G, Fanek Z, Greco DJ, Wu PM, Doykan CE, Kiner O, Lawson RJ, Frosch MP, Pochet N, Fatimy RE, Krichevsky AM, Gygi SP, Lassmann H, Berry J, Cudkowicz ME, Weiner HL (2015) Targeting miR-155 restores abnormal microglia and attenuates disease in SOD1 mice. Ann Neurol 77 (1):75-99. doi:10.1002/ana.24304

23. Ben Haim L, Carrillo-de Sauvage MA, Ceyzeriat K, Escartin C (2015) Elusive roles for reactive astrocytes in neurodegenerative diseases. Front Cell Neurosci 9:278. doi:10.3389/fncel.2015.00278

24. Sison SL, Patitucci TN, Seminary ER, Villalon E, Lorson CL, Ebert AD (2017) Astrocyteproduced miR-146a as a mediator of motor neuron loss in spinal muscular atrophy. Hum Mol Genet 26 (17):3409-3420. doi:10.1093/hmg/ddx230

25. Trias E, Ibarburu S, Barreto-Nunez R, Barbeito L (2017) Significance of aberrant glial cell phenotypes in pathophysiology of amyotrophic lateral sclerosis. Neurosci Lett 636:27-31. doi:S0304-3940(16)30546-8 [pii]

10.1016/j.neulet.2016.07.052

26. Rocha MC, Pousinha PA, Correia AM, Sebastiao AM, Ribeiro JA (2013) Early changes of neuromuscular transmission in the SOD1(G93A) mice model of ALS start long before motor symptoms onset. PLoS One 8 (9):e73846. doi:10.1371/journal.pone.0073846

27. Nascimento F, Pousinha PA, Correia AM, Gomes R, Sebastiao AM, Ribeiro JA (2014) Adenosine A2A receptors activation facilitates neuromuscular transmission in the presymptomatic phase of the SOD1(G93A) ALS mice, but not in the symptomatic phase. PLoS One 9 (8):e104081. doi:10.1371/journal.pone.0104081

PONE-D-14-14797 [pii]

28. Fernandes A, Barateiro A, Falcão AS, Silva SL, Vaz AR, Brito MA, Silva RF, Brites D (2011) Astrocyte reactivity to unconjugated bilirubin requires TNF-alpha and IL-1beta receptor signaling pathways. Glia 59 (1):14-25. doi:10.1002/glia.21072

29. Vaz AR, Cunha C, Gomes C, Schmucki N, Barbosa M, Brites D (2015) Glycoursodeoxycholic acid reduces matrix metalloproteinase- 9 and caspase- 9 activation in a cellular model of superoxide dismutase-1 neurodegeneration. Mol Neurobiol 51 (3):864-877. doi:10.1007/s12035-014-8731-8

30. Phatnani HP, Guarnieri P, Friedman BA, Carrasco MA, Muratet M, O'Keeffe S, Nwakeze C, Pauli-Behn F, Newberry KM, Meadows SK, Tapia JC, Myers RM, Maniatis T (2013) Intricate 
interplay between astrocytes and motor neurons in ALS. Proc Natl Acad Sci U S A 110 (8):E756765. doi:10.1073/pnas.1222361110

31. Wang L, Popko B, Roos RP (2014) An enhanced integrated stress response ameliorates mutant SOD1-induced ALS. Hum Mol Genet 23 (10):2629-2638. doi:10.1093/hmg/ddt658

ddt658 [pii]

32. Kim HJ, Raphael AR, LaDow ES, McGurk L, Weber RA, Trojanowski JQ, Lee VM, Finkbeiner S, Gitler AD, Bonini NM (2014) Therapeutic modulation of elF2alpha phosphorylation rescues TDP-43 toxicity in amyotrophic lateral sclerosis disease models. Nat Genet 46 (2):152-160. doi:10.1038/ng.2853

ng.2853 [pii]

33. B'Chir W, Maurin AC, Carraro V, Averous J, Jousse C, Muranishi Y, Parry L, Stepien G, Fafournoux P, Bruhat A (2013) The elF2alpha/ATF4 pathway is essential for stress-induced autophagy gene expression. Nucleic Acids Res 41 (16):7683-7699. doi:10.1093/nar/gkt563

gkt563 [pii]

34. Sakowski SA, Lunn JS, Busta AS, Oh SS, Zamora-Berridi G, Palmer M, Rosenberg AA, Philip SG, Dowling JJ, Feldman EL (2012) Neuromuscular effects of G93A-SOD1 expression in zebrafish. Mol Neurodegener 7:44. doi:10.1186/1750-1326-7-44

35. Pasini S, Corona C, Liu J, Greene LA, Shelanski ML (2015) Specific Downregulation of Hippocampal ATF4 Reveals a Necessary Role in Synaptic Plasticity and Memory. Cell Reports 11 (2):183-191. doi:10.1016/j.celrep.2015.03.025

36. Matus S, Lopez E, Valenzuela V, Nassif M, Hetz C (2013) Functional contribution of the transcription factor ATF4 to the pathogenesis of amyotrophic lateral sclerosis. PLoS One 8 (7):e66672. doi:10.1371/journal.pone.0066672

37. Arber S, Han B, Mendelsohn M, Smith M, Jessell TM, Sockanathan S (1999) Requirement for the homeobox gene $\mathrm{Hb9}$ in the consolidation of motor neuron identity. Neuron 23 (4):659674. doi:S0896-6273(01)80026-X [pii]

38. Savioz A, Leuba G, Vallet PG (2014) A framework to understand the variations of PSD-95 expression in brain aging and in Alzheimer's disease. Ageing Res Rev 18:86-94. doi:10.1016/j.arr.2014.09.004

S1568-1637(14)00107-X [pii]

39. Hou Q, Ruan H, Gilbert J, Wang G, Ma Q, Yao WD, Man HY (2015) MicroRNA miR124 is required for the expression of homeostatic synaptic plasticity. Nat Commun 6:10045. doi:10.1038/ncomms10045

ncomms10045 [pii]

40. Le MT, Xie H, Zhou B, Chia PH, Rizk P, Um M, Udolph G, Yang H, Lim B, Lodish HF (2009) MicroRNA-125b promotes neuronal differentiation in human cells by repressing multiple targets. Mol Cell Biol 29 (19):5290-5305. doi:10.1128/MCB.01694-08

MCB.01694-08 [pii]

41. Frakes AE, Ferraiuolo L, Haidet-Phillips AM, Schmelzer L, Braun L, Miranda CJ, Ladner KJ, Bevan AK, Foust KD, Godbout JP, Popovich PG, Guttridge DC, Kaspar BK (2014) Microglia induce motor neuron death via the classical NF-kappaB pathway in amyotrophic lateral sclerosis. Neuron 81 (5):1009-1023. doi:10.1016/j.neuron.2014.01.013

42. Kaltschmidt B, Kaltschmidt C (2009) NF-kappa B in the Nervous System. Cold Spring Harbor Perspectives in Biology 1 (3). doi:ARTN a001271

10.1101/cshperspect.a001271 
43. Shih RH, Wang CY, Yang CM (2015) NF-kappaB Signaling Pathways in Neurological Inflammation: A Mini Review. Frontiers in Molecular Neuroscience 8. doi:Artn 77

10.3389/Fnmol.2015.00077

44. Luan ZG, Zhang H, Yang PT, Ma XC, Zhang C, Guo RX (2010) HMGB1 activates nuclear factor-kappaB signaling by RAGE and increases the production of TNF-alpha in human umbilical vein endothelial cells. Immunobiology 215 (12):956-962. doi:10.1016/j.imbio.2009.11.001

S0171-2985(09)00171-5 [pii]

45. Lo Coco D, Veglianese P, Allievi E, Bendotti C (2007) Distribution and cellular localization of high mobility group box protein 1 (HMGB1) in the spinal cord of a transgenic mouse model of ALS. Neuroscience Letters 412 (1):73-77. doi:10.1016/j.neulet.2006.10.063

46. Bohlig L, Rother K (2011) One function--multiple mechanisms: the manifold activities of p53 as a transcriptional repressor. J Biomed Biotechnol 2011:464916. doi:10.1155/2011/464916

47. Volonte C, Apolloni S, Parisi C (2015) MicroRNAs: newcomers into the ALS picture. CNS Neurol Disord Drug Targets 14 (2):194-207

48. Ma X, Becker Buscaglia LE, Barker JR, Li Y (2011) MicroRNAs in NF-kappaB signaling. J Mol Cell Biol 3 (3):159-166. doi:10.1093/jmcb/mjr007

mjr007 [pii]

49. Iyer A, Zurolo E, Prabowo A, Fluiter K, Spliet WG, van Rijen PC, Gorter JA, Aronica E (2012) MicroRNA-146a: a key regulator of astrocyte-mediated inflammatory response. PLoS One 7 (9):e44789. doi:10.1371/journal.pone.0044789

50. Bunton-Stasyshyn RK, Saccon RA, Fratta P, Fisher EM (2015) SOD1 Function and Its Implications for Amyotrophic Lateral Sclerosis Pathology: New and Renascent Themes. Neuroscientist 21 (5):519-529. doi:10.1177/1073858414561795

1073858414561795 [pii]

51. Ferraiuolo L, Meyer K, Sherwood TW, Vick J, Likhite S, Frakes A, Miranda CJ, Braun L, Heath PR, Pineda R, Beattie CE, Shaw PJ, Askwith CC, McTigue D, Kaspar BK (2016) Oligodendrocytes contribute to motor neuron death in ALS via SOD1-dependent mechanism. Proc Natl Acad Sci U S A 113 (42):E6496-E6505. doi:1607496113 [pii]

10.1073/pnas.1607496113

52. Philips T, Bento-Abreu A, Nonneman A, Haeck W, Staats K, Geelen V, Hersmus N, Kusters B, Van Den Bosch L, Van Damme P, Richardson WD, Robberecht W (2013) Oligodendrocyte dysfunction in the pathogenesis of amyotrophic lateral sclerosis. Brain 136 (Pt 2):471-482. doi:10.1093/brain/aws339

53. Nikodemova M, Small AL, Smith SMC, Mitchell GS, Watters JJ (2014) Spinal but not cortical microglia acquire an atypical phenotype with high VEGF, galectin-3 and osteopontin, and blunted inflammatory responses in ALS rats23. Neurobiology of Disease 69:43-53. doi:10.1016/j.nbd.2013.11.009

54. Cardoso AL, Guedes JR, Pereira de Almeida L, Pedroso de Lima MC (2012) miR-155 modulates microglia-mediated immune response by down-regulating SOCS-1 and promoting cytokine and nitric oxide production. Immunology 135 (1):73-88. doi:10.1111/j.13652567.2011.03514.x

55. Haidet-Phillips AM, Hester ME, Miranda CJ, Meyer K, Braun L, Frakes A, Song S, Likhite S, Murtha MJ, Foust KD, Rao M, Eagle A, Kammesheidt A, Christensen A, Mendell JR, Burghes AH, Kaspar BK (2011) Astrocytes from familial and sporadic ALS patients are toxic to motor neurons. Nat Biotechnol 29 (9):824-828. doi:10.1038/nbt.1957 
56. Theodoric N, Bechberger JF, Naus CC, Sin WC (2012) Role of gap junction protein connexin43 in astrogliosis induced by brain injury. PLoS One 7 (10):e47311. doi:10.1371/journal.pone.0047311

PONE-D-12-14227 [pii]

57. Serrano A, Donno C, Giannetti S, Peric M, Andjus P, D'Ambrosi N, Michetti F (2017) The Astrocytic S100B Protein with Its Receptor RAGE Is Aberrantly Expressed in SOD1(G93A) Models, and Its Inhibition Decreases the Expression of Proinflammatory Genes. Mediators Inflamm 2017:1626204. doi:10.1155/2017/1626204

58. Tian R, Wu X, Hagemann TL, Sosunov AA, Messing A, McKhann GM, Goldman JE (2010) Alexander disease mutant glial fibrillary acidic protein compromises glutamate transport in astrocytes. J Neuropathol Exp Neurol 69 (4):335-345. doi:10.1097/NEN.0b013e3181d3cb52

00005072-201004000-00002 [pii]

59. Lepekhin EA, Eliasson C, Berthold CH, Berezin V, Bock E, Pekny M (2001) Intermediate filaments regulate astrocyte motility. J Neurochem 79 (3):617-625

60. Yoshii Y, Otomo A, Pan L, Ohtsuka M, Hadano S (2011) Loss of glial fibrillary acidic protein marginally accelerates disease progression in a SOD1(H46R) transgenic mouse model of ALS. Neurosci Res 70 (3):321-329. doi:10.1016/j.neures.2011.03.006

61. Baker DJ, Blackburn DJ, Keatinge M, Sokhi D, Viskaitis P, Heath PR, Ferraiuolo L, Kirby J, Shaw PJ (2015) Lysosomal and phagocytic activity is increased in astrocytes during disease progression in the SOD1 (G93A) mouse model of amyotrophic lateral sclerosis. Front Cell Neurosci 9:410. doi:10.3389/fncel.2015.00410

62. Sidoryk-Wegrzynowicz M, Gerber YN, Ries M, Sastre M, Tolkovsky AM, Spillantini MG (2017) Astrocytes in mouse models of tauopathies acquire early deficits and lose neurosupportive functions. Acta Neuropathol Commun 5 (1):89. doi:10.1186/s40478-0170478-9

10.1186/s40478-017-0478-9 [pii]

63. Li M, Sun L, Luo Y, Xie C, Pang Y, Li Y (2014) High-mobility group box 1 released from astrocytes promotes the proliferation of cultured neural stem/progenitor cells. Int J Mol Med 34 (3):705-714. doi:10.3892/ijmm.2014.1820

64. Xie ZF, Xin G, Xu YX, SU Y, Li KS (2016) LPS-Primed Release of HMGB-1 from Cortical Astrocytes is Modulated Through PI3K/AKT Pathway. Cell Mol Neurobiol 36 (1):93-102. doi:10.1007/s10571-015-0223-5

10.1007/s10571-015-0223-5 [pii]

65. Pedrazzi M, Patrone M, Passalacqua M, Ranzato E, Colamassaro D, Sparatore B, Pontremoli S, Melloni E (2007) Selective proinflammatory activation of astrocytes by high-mobility group box 1 protein signaling. J Immunol 179 (12):8525-8532. doi:179/12/8525 [pii]

66. Bhalala OG, Pan L, Sahni V, McGuire TL, Gruner K, Tourtellotte WG, Kessler JA (2012) microRNA-21 regulates astrocytic response following spinal cord injury. J Neurosci 32 (50):17935-17947. doi:10.1523/JNEUROSCI.3860-12.2012

67. Strickland ER, Hook MA, Balaraman S, Huie JR, Grau JW, Miranda RC (2011) MicroRNA dysregulation following spinal cord contusion: implications for neural plasticity and repair. Neuroscience 186:146-160. doi:10.1016/j.neuroscience.2011.03.063

S0306-4522(11)00371-X [pii]

68. Qian K, Huang H, Peterson A, Hu B, Maragakis NJ, Ming GL, Chen H, Zhang SC (2017) Sporadic ALS Astrocytes Induce Neuronal Degeneration In Vivo. Stem Cell Reports 8 (4):843855. doi:S2213-6711(17)30091-7 [pii]

10.1016/j.stemcr.2017.03.003 
69. Tripathi P, Rodriguez-Muela N, Klim JR, de Boer AS, Agrawal S, Sandoe J, Lopes CS, Ogliari KS, Williams LA, Shear M, Rubin LL, Eggan K, Zhou Q (2017) Reactive Astrocytes Promote ALSlike Degeneration and Intracellular Protein Aggregation in Human Motor Neurons by Disrupting Autophagy through TGF-beta1. Stem Cell Reports 9 (2):667-680. doi:S22136711(17)30271-0 [pii]

10.1016/j.stemcr.2017.06.008

70. Casas C, Manzano R, Vaz AR, Rosario O, Brites D (2016) Synaptic Failure: Focus in an Integrative View of ALS Brain Plasticity 1 (2):159-175

71. Thomsen GM, Gowing G, Latter J, Chen M, Vit JP, Staggenborg K, Avalos P, Alkaslasi M, Ferraiuolo L, Likhite S, Kaspar BK, Svendsen CN (2014) Delayed disease onset and extended survival in the SOD1G93A rat model of amyotrophic lateral sclerosis after suppression of mutant SOD1 in the motor cortex. J Neurosci 34 (47):15587-15600. doi:10.1523/JNEUROSCI.2037-14.2014

34/47/15587 [pii]

72. Wang J, Xu G, Borchelt DR (2002) High molecular weight complexes of mutant superoxide dismutase 1: age-dependent and tissue-specific accumulation. Neurobiol Dis 9 (2):139-148. doi:10.1006/nbdi.2001.0471

S0969996101904711 [pii]

73. Wang $M, X u Q$, Yuan $M(2011)$ The unfolded protein response induced by salt stress in Arabidopsis. Methods Enzymol 489:319-328. doi:10.1016/B978-0-12-385116-1.00018-2

B978-0-12-385116-1.00018-2 [pii]

74. Saxena S, Cabuy E, Caroni P (2009) A role for motoneuron subtype-selective ER stress in disease manifestations of FALS mice. Nat Neurosci 12 (5):627-636. doi:10.1038/nn.2297

75. Yip PK, Pizzasegola C, Gladman S, Biggio ML, Marino M, Jayasinghe M, Ullah F, Dyall SC, Malaspina A, Bendotti C, Michael-Titus A (2013) The omega-3 fatty acid eicosapentaenoic acid accelerates disease progression in a model of amyotrophic lateral sclerosis. PLoS One 8 (4):e61626. doi:10.1371/journal.pone.0061626

PONE-D-13-01360 [pii]

76. Genc B, Jara JH, Lagrimas AK, Pytel P, Roos RP, Mesulam MM, Geula C, Bigio EH, Ozdinler PH (2017) Apical dendrite degeneration, a novel cellular pathology for Betz cells in ALS. Sci Rep 7:41765. doi:10.1038/srep41765

srep41765 [pii]

77. Cruz-Sanchez FF, Moral A, Rossi ML, Quinto L, Castejon C, Tolosa E, de Belleroche J (1996) Synaptophysin in spinal anterior horn in aging and ALS: an immunohistological study. J Neural Transm (Vienna) 103 (11):1317-1329. doi:10.1007/BF01271192 [pii]

10.1007/BF01271192

78. Abe M, Bonini NM (2013) MicroRNAs and neurodegeneration: role and impact. Trends Cell Biol 23 (1):30-36. doi:10.1016/j.tcb.2012.08.013

79. Zeng Y (2009) Regulation of the mammalian nervous system by microRNAs. Mol Pharmacol 75 (2):259-264. doi:10.1124/mol.108.052118

mol.108.052118 [pii]

80. Quiroz JFD, Tsai E, Coyle M, Sehm T, Echeverri K (2014) Precise control of miR-125b levels is required to create a regeneration-permissive environment after spinal cord injury: a crossspecies comparison between salamander and rat. Disease Models \& Mechanisms 7 (6):601611. doi:10.1242/dmm.014837 
81. Zhou F, Zhang C, Guan Y, Chen Y, Lu Q, Jie L, Gao H, Du H, Zhang H, Liu Y, Wang X (2017) Screening the expression characteristics of several miRNAs in G93A-SOD1 transgenic mouse: altered expression of miRNA-124 is associated with astrocyte differentiation by targeting Sox2 and Sox9. J Neurochem:doi: 10.1111/jnc.14229. doi:10.1111/jnc.14229

82. Campos-Melo D, Droppelmann CA, He Z, Volkening K, Strong MJ (2013) Altered microRNA expression profile in Amyotrophic Lateral Sclerosis: a role in the regulation of NFL mRNA levels. Mol Brain 6:26. doi:10.1186/1756-6606-6-26

83. Benigni M, Ricci C, Jones AR, Giannini F, Al-Chalabi A, Battistini S (2016) Identification of miRNAs as Potential Biomarkers in Cerebrospinal Fluid from Amyotrophic Lateral Sclerosis Patients. Neuromolecular Med 18 (4):551-560. doi:10.1007/s12017-016-8396-8

84. Casula M, Iyer AM, Spliet WG, Anink JJ, Steentjes K, Sta M, Troost D, Aronica E (2011) Tolllike receptor signaling in amyotrophic lateral sclerosis spinal cord tissue. Neuroscience 179:233-243. doi:S0306-4522(11)00116-3 [pii]

10.1016/j.neuroscience.2011.02.001

85. Crosio C, Valle C, Casciati A, laccarino C, Carri MT (2011) Astroglial inhibition of NF-kappaB does not ameliorate disease onset and progression in a mouse model for amyotrophic lateral sclerosis (ALS). PLoS One 6 (3):e17187. doi:10.1371/journal.pone.0017187

86. Liu Z, Li Y, Cui Y, Roberts C, Lu M, Wilhelmsson U, Pekny M, Chopp M (2014) Beneficial effects of gfap/vimentin reactive astrocytes for axonal remodeling and motor behavioral recovery in mice after stroke. Glia 62 (12):2022-2033. doi:10.1002/glia.22723

87. Kunze A, Lengacher S, Dirren E, Aebischer P, Magistretti PJ, Renaud P (2013) Astrocyteneuron co-culture on microchips based on the model of SOD mutation to mimic ALS. Integr Biol (Camb) 5 (7):964-975. doi:10.1039/c3ib40022k

88. Foran E, Trotti D (2009) Glutamate transporters and the excitotoxic path to motor neuron degeneration in amyotrophic lateral sclerosis. Antioxid Redox Signal 11 (7):1587-1602. doi:10.1089/ars.2009.2444

89. Mei J, Bachoo R, Zhang CL (2011) MicroRNA-146a inhibits glioma development by targeting Notch1. Mol Cell Biol 31 (17):3584-3592. doi:10.1128/MCB.05821-11

MCB.05821-11 [pii]

90. Zhang QB, Qing YF, Yin CC, Zhou L, Liu XS, Mi QS, Zhou JG (2018) Mice with miR-146a deficiency develop severe gouty arthritis via dysregulation of TRAF 6, IRAK 1 and NALP3 inflammasome. Arthritis Res Ther 20 (1):45. doi:10.1186/s13075-018-1546-7

91. Taganov KD, Boldin MP, Chang KJ, Baltimore D (2006) NF-kappaB-dependent induction of microRNA miR-146, an inhibitor targeted to signaling proteins of innate immune responses. Proc Natl Acad Sci U S A 103 (33):12481-12486. doi:0605298103 [pii]

$10.1073 /$ pnas. 0605298103

92. Lakatos A (2017) State-of-art modelling of inflammatory astrocyte-synapse interactions in injury and amyotrophic lateral sclerosis. Neural Regen Res 12 (1):75-76. doi:10.4103/16735374.198977

NRR-12-75 [pii]

93. Kaplan A, Spiller KJ, Towne C, Kanning KC, Choe GT, Geber A, Akay T, Aebischer P, Henderson CE (2014) Neuronal matrix metalloproteinase-9 is a determinant of selective neurodegeneration. Neuron 81 (2):333-348. doi:10.1016/j.neuron.2013.12.009

S0896-6273(13)01139-2 [pii]

94. Fang P, Schachner M, Shen YQ (2012) HMGB1 in development and diseases of the central nervous system. Mol Neurobiol 45 (3):499-506. doi:10.1007/s12035-012-8264-y 
Figure 1

a
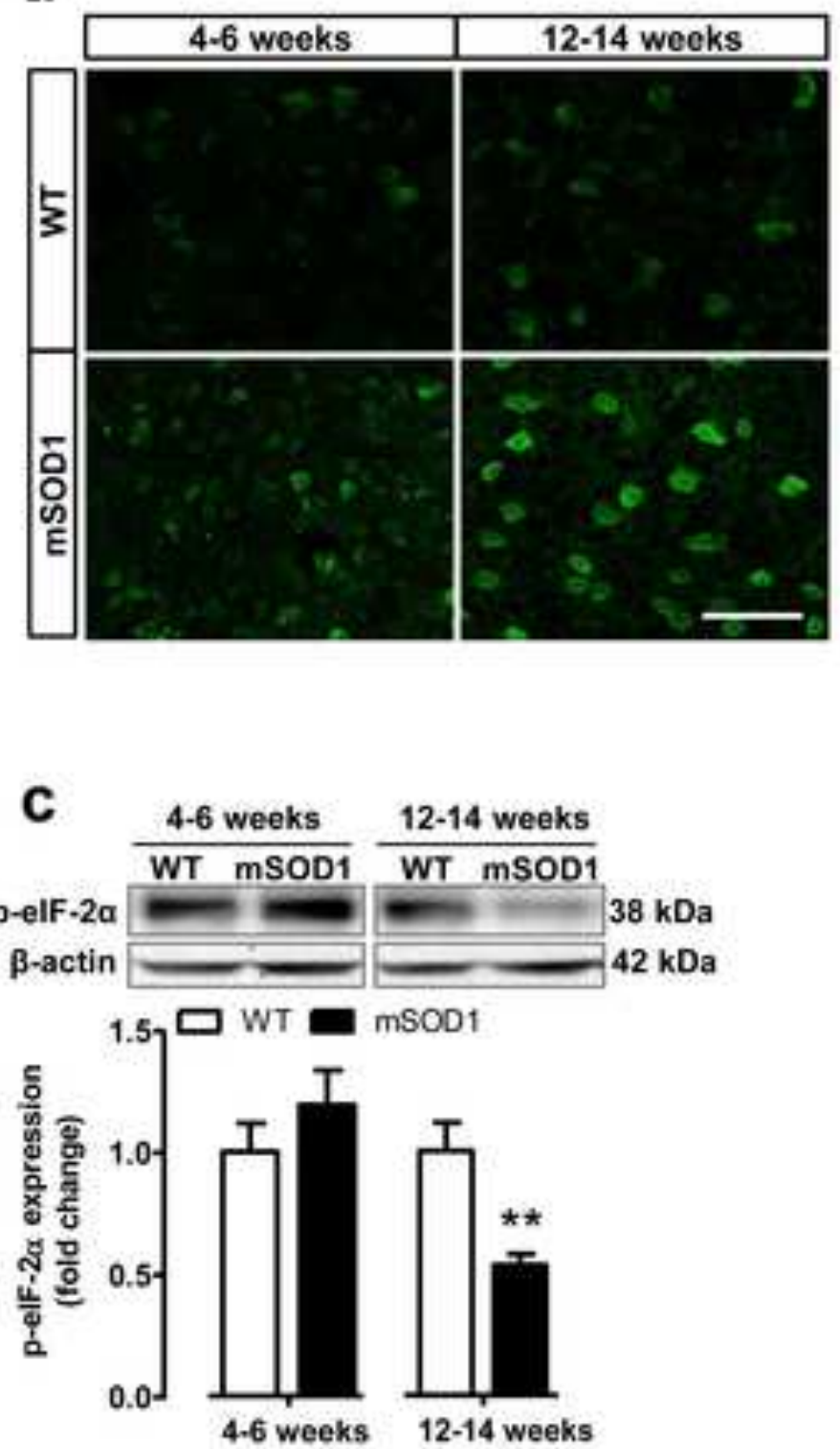
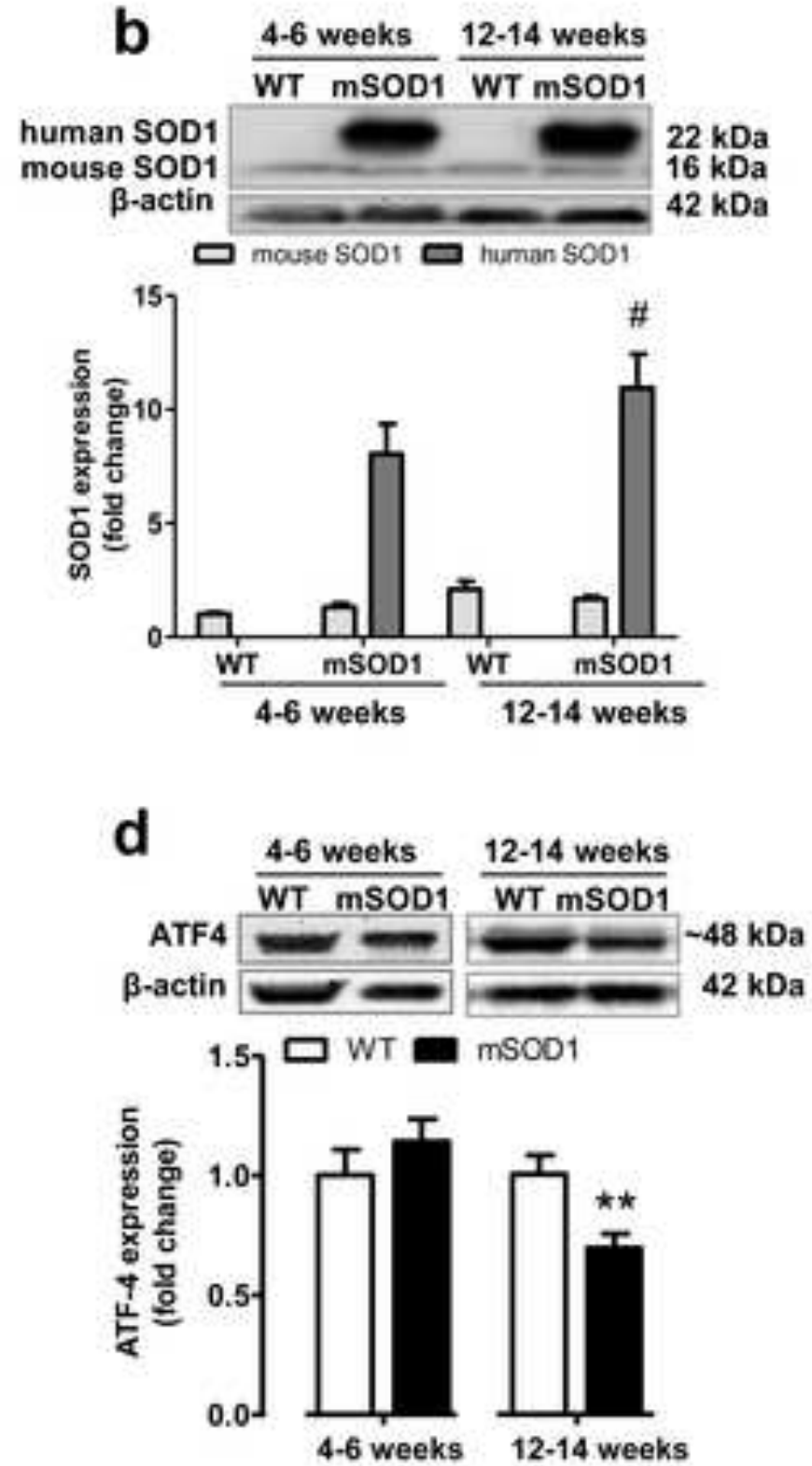

Gomes C. et al. 
Figure 2
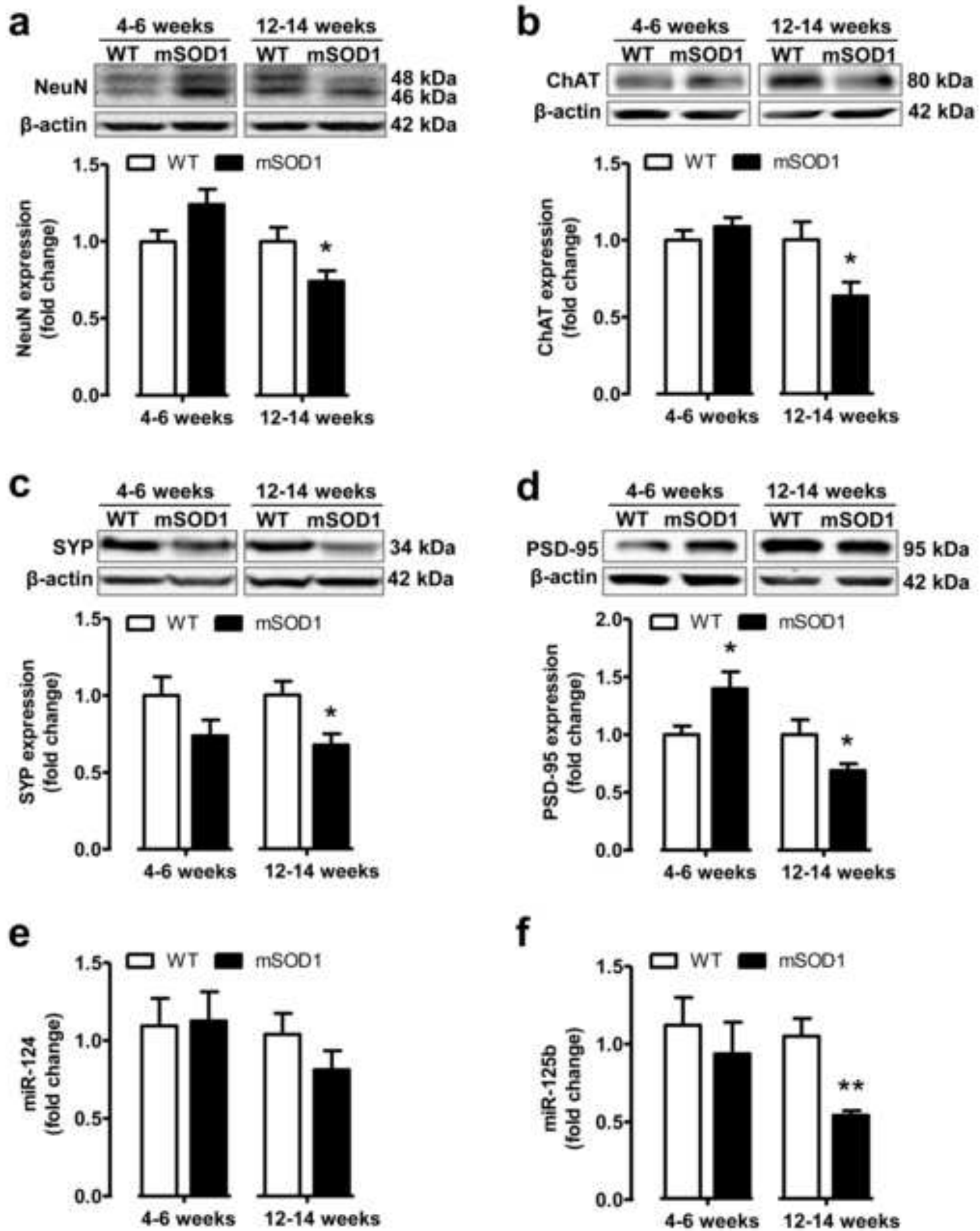

Gomes C. et al. 


\section{Figure 3}
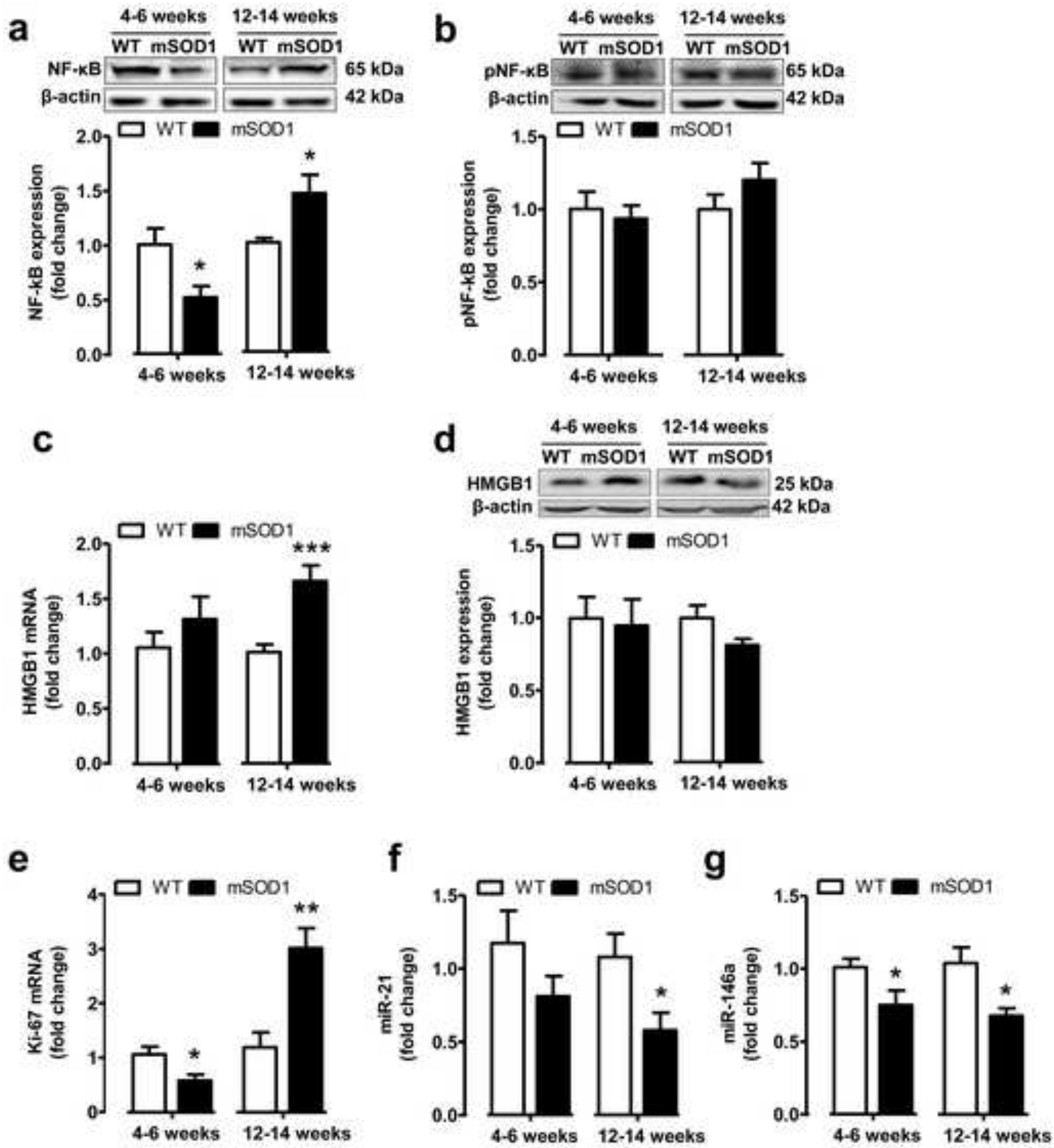

Gomes C. et al. 
Figure 4
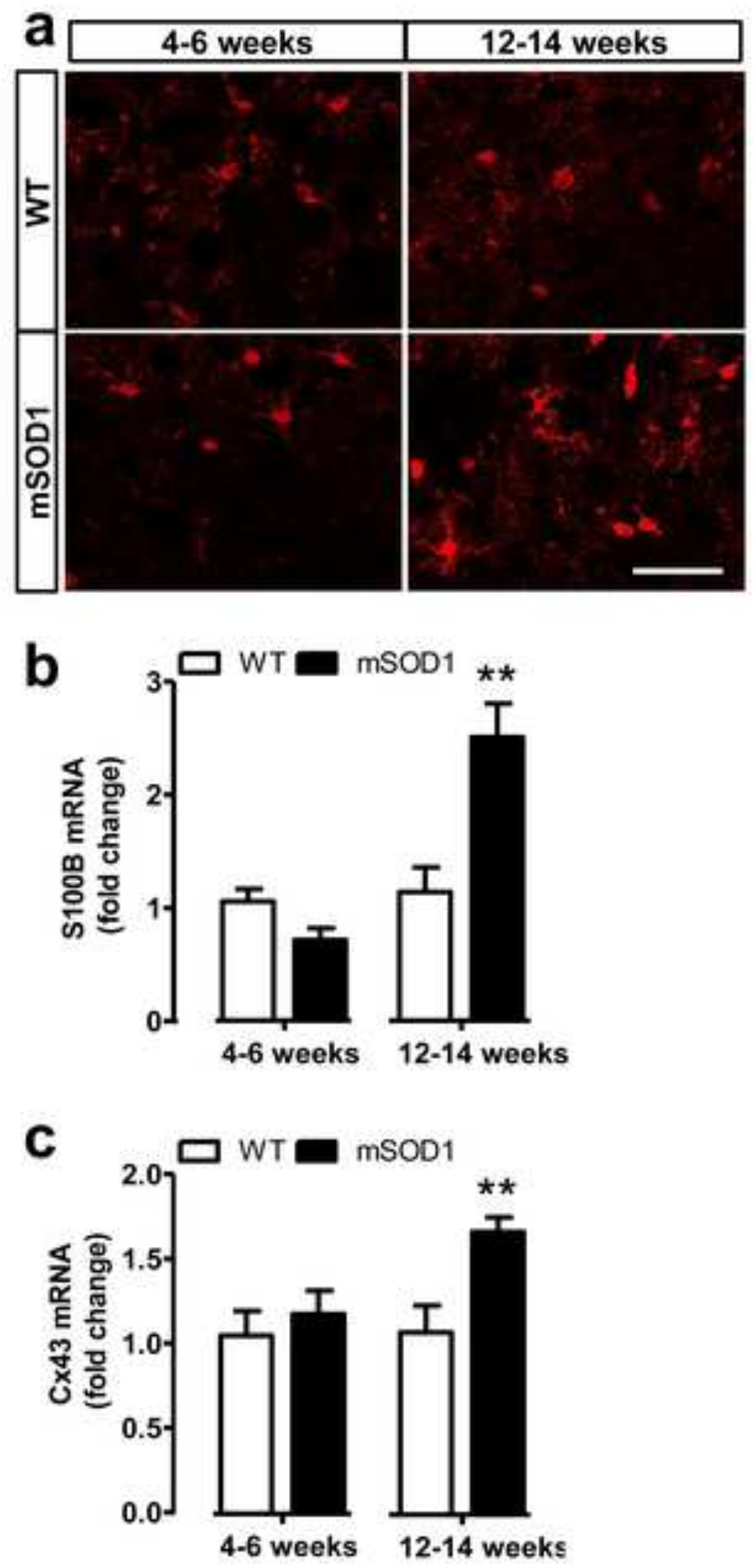

Gomes C. et al. 
Figure 5
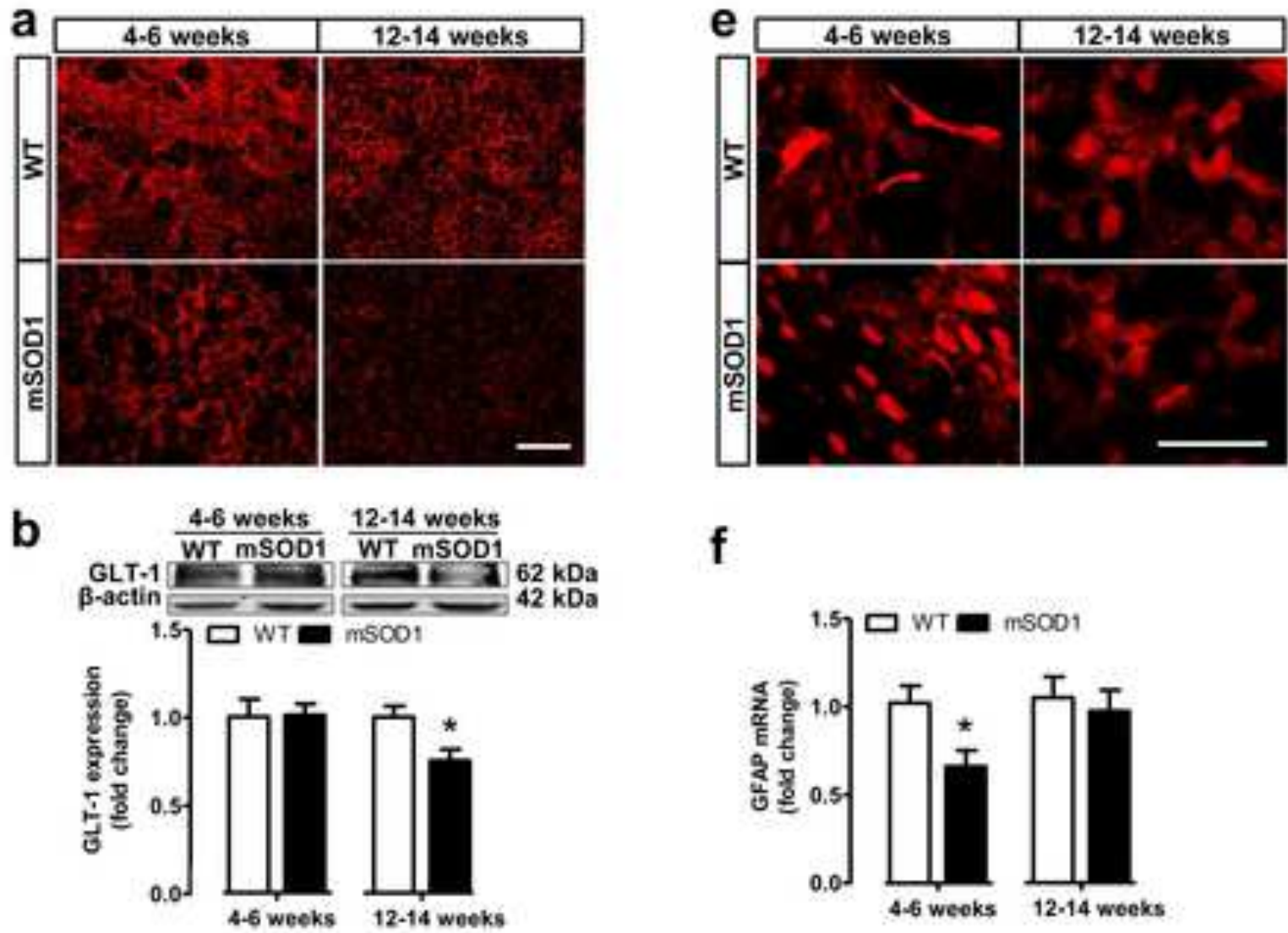

f

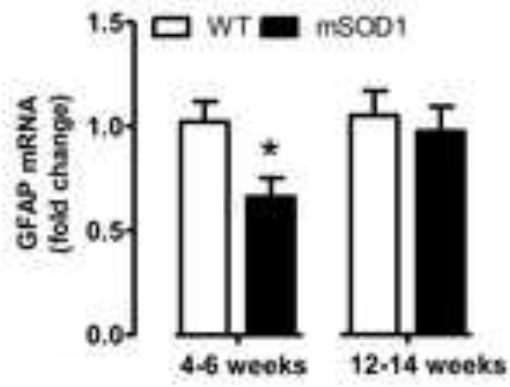

C
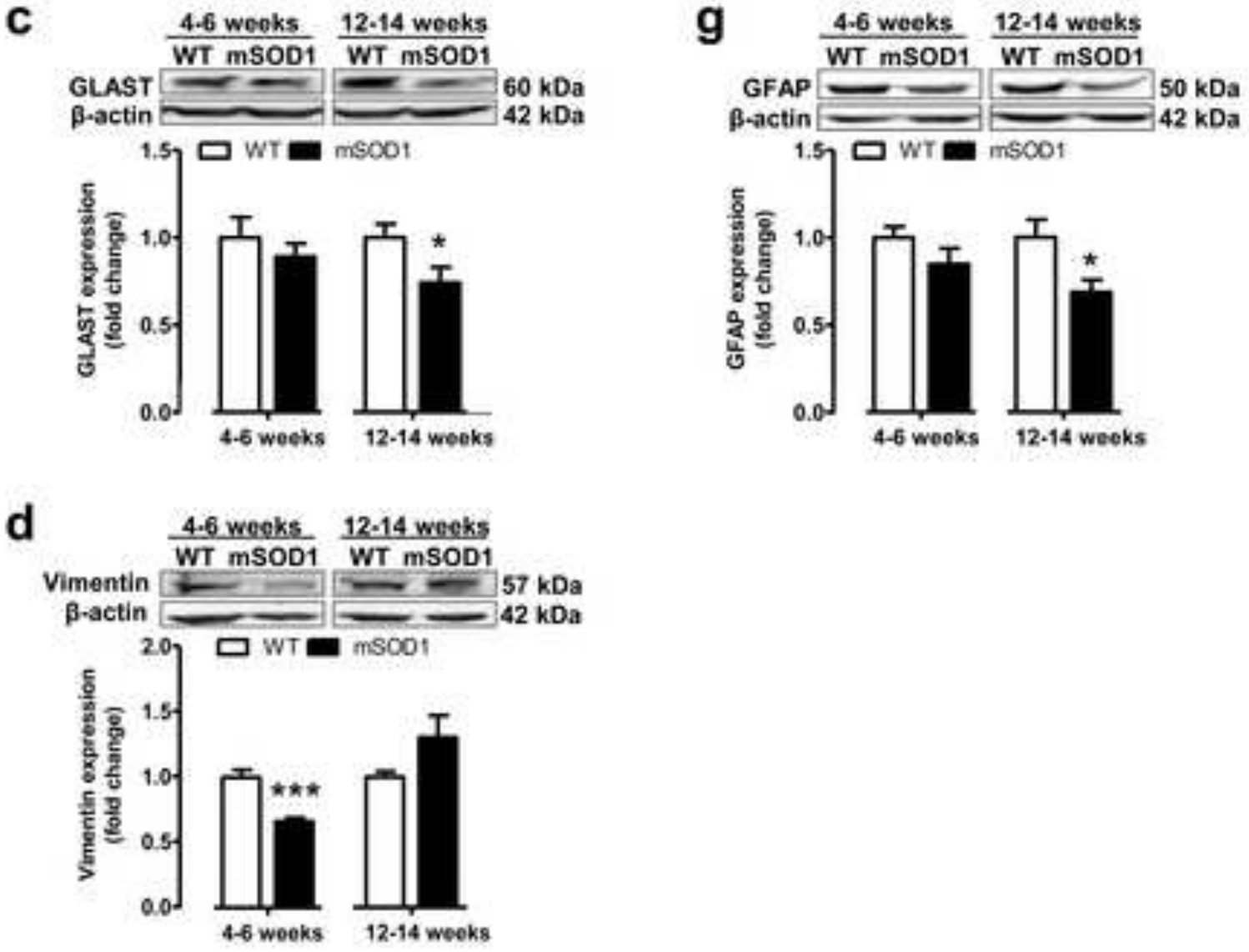

Gomes C. et al. 
Figure 6

a
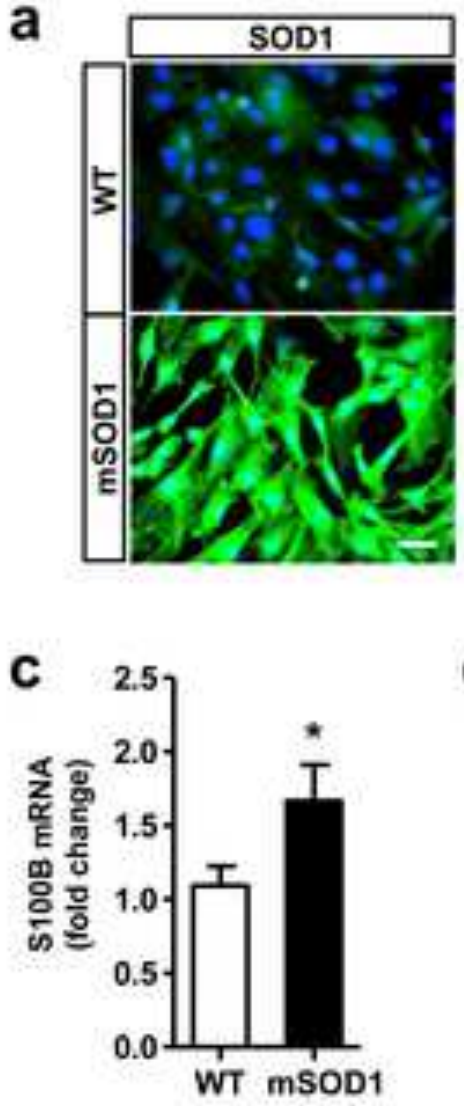

W WT mSOD1
GFAP $-50 \mathrm{kDa}$
$\beta$-actin $-42 \mathrm{kDa}$

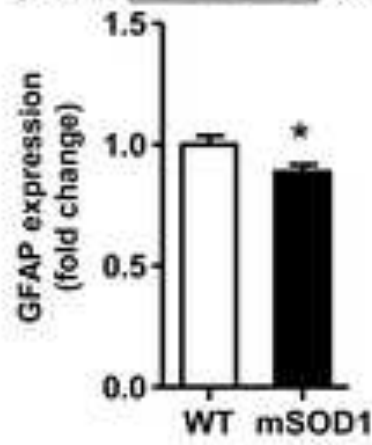

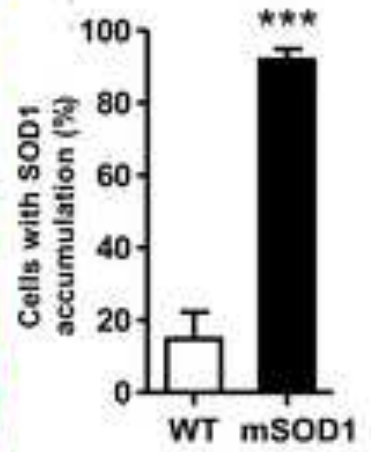
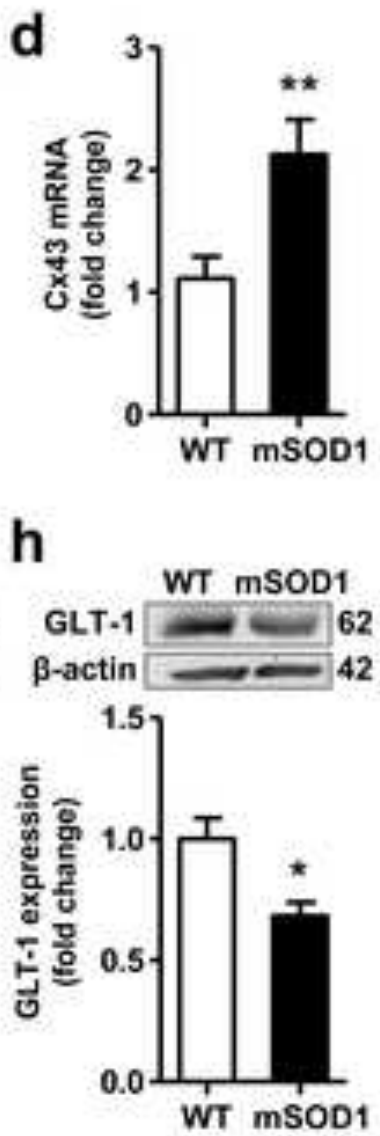

b
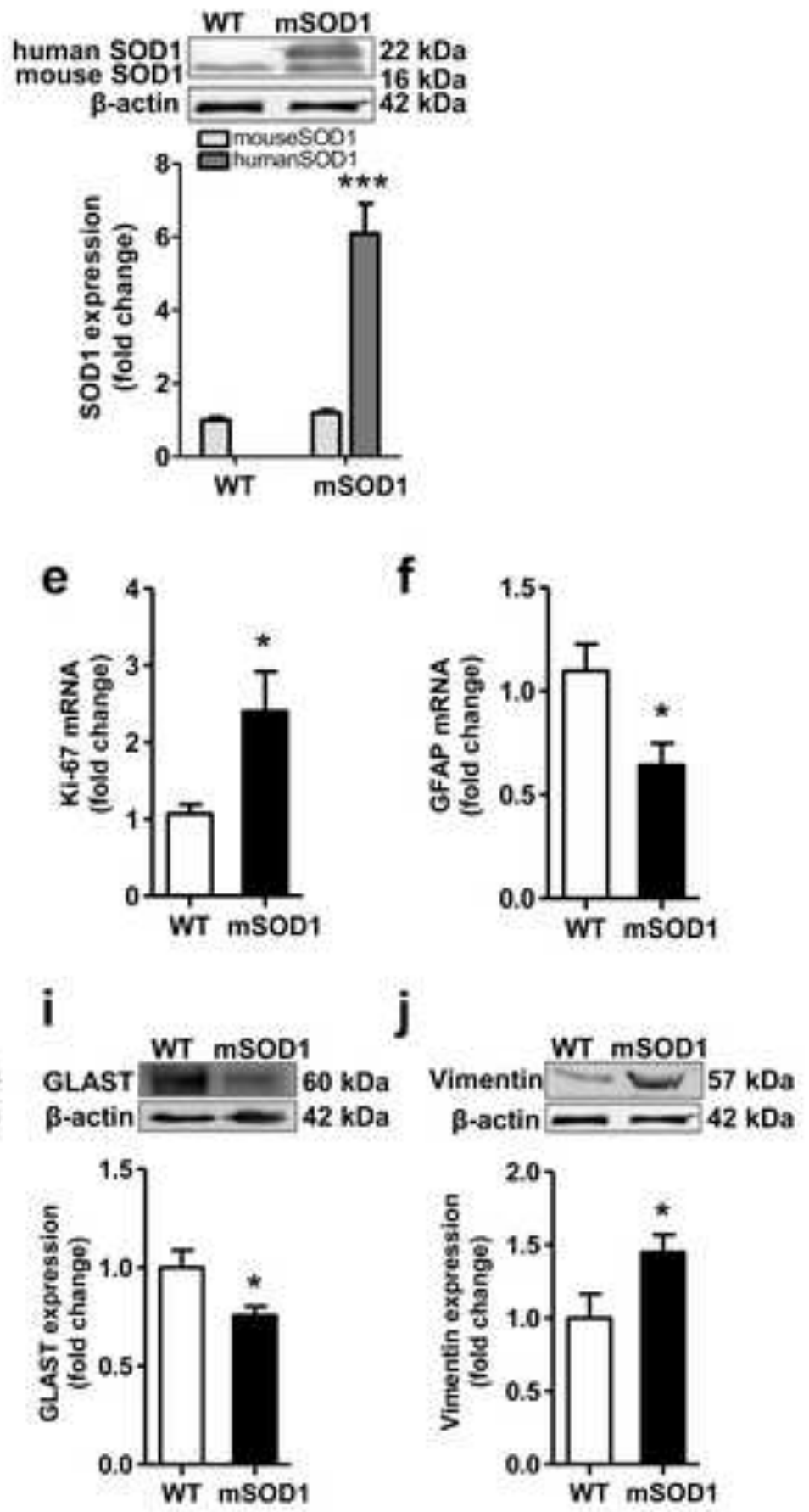

Gomes C. et al. 
Figure 7

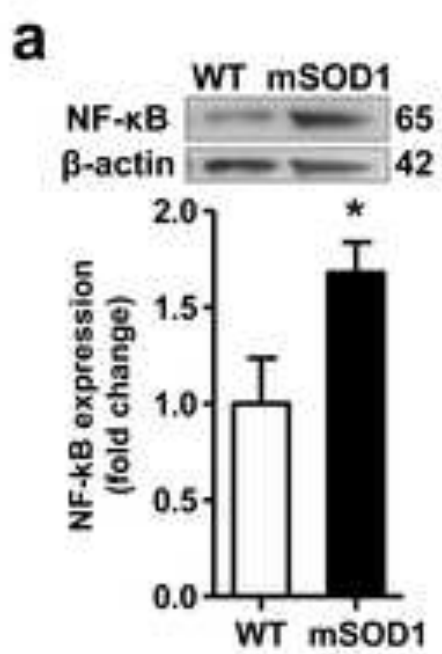

b
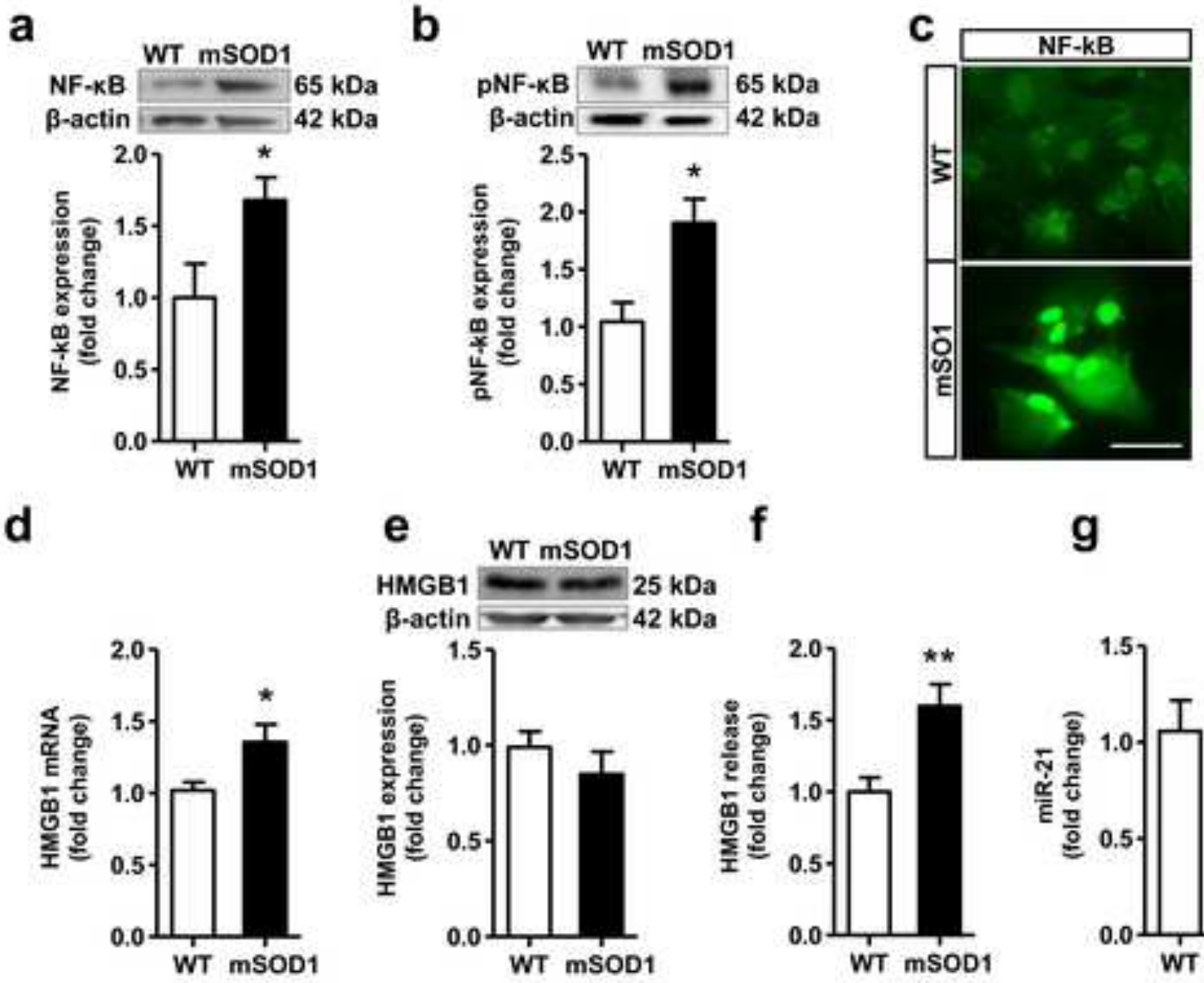

g
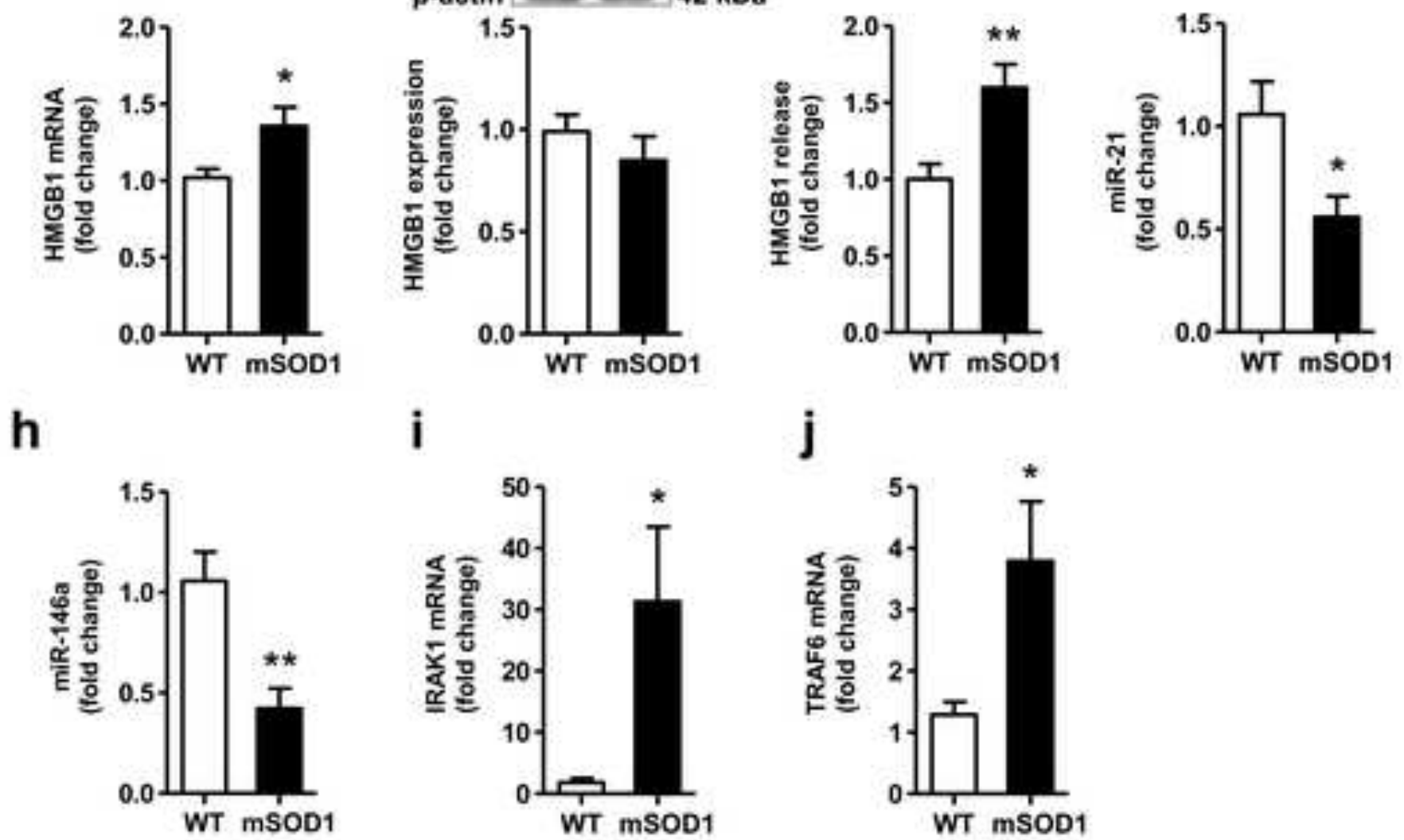

Gomes C. et al. 
Figure 8
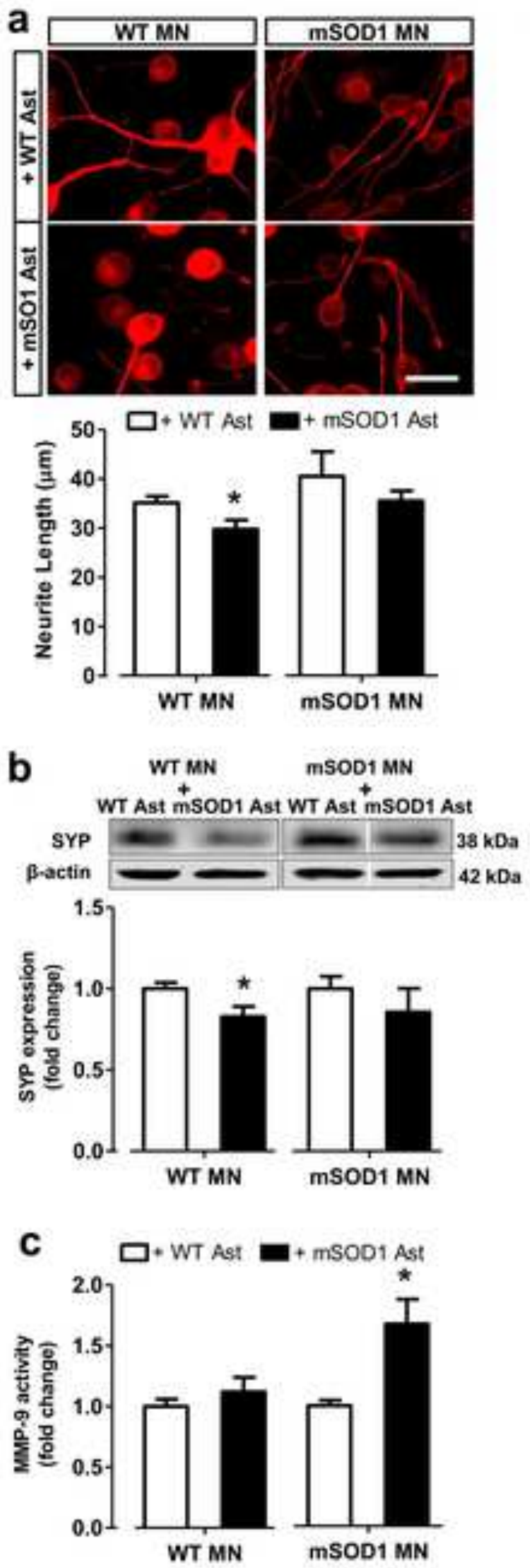
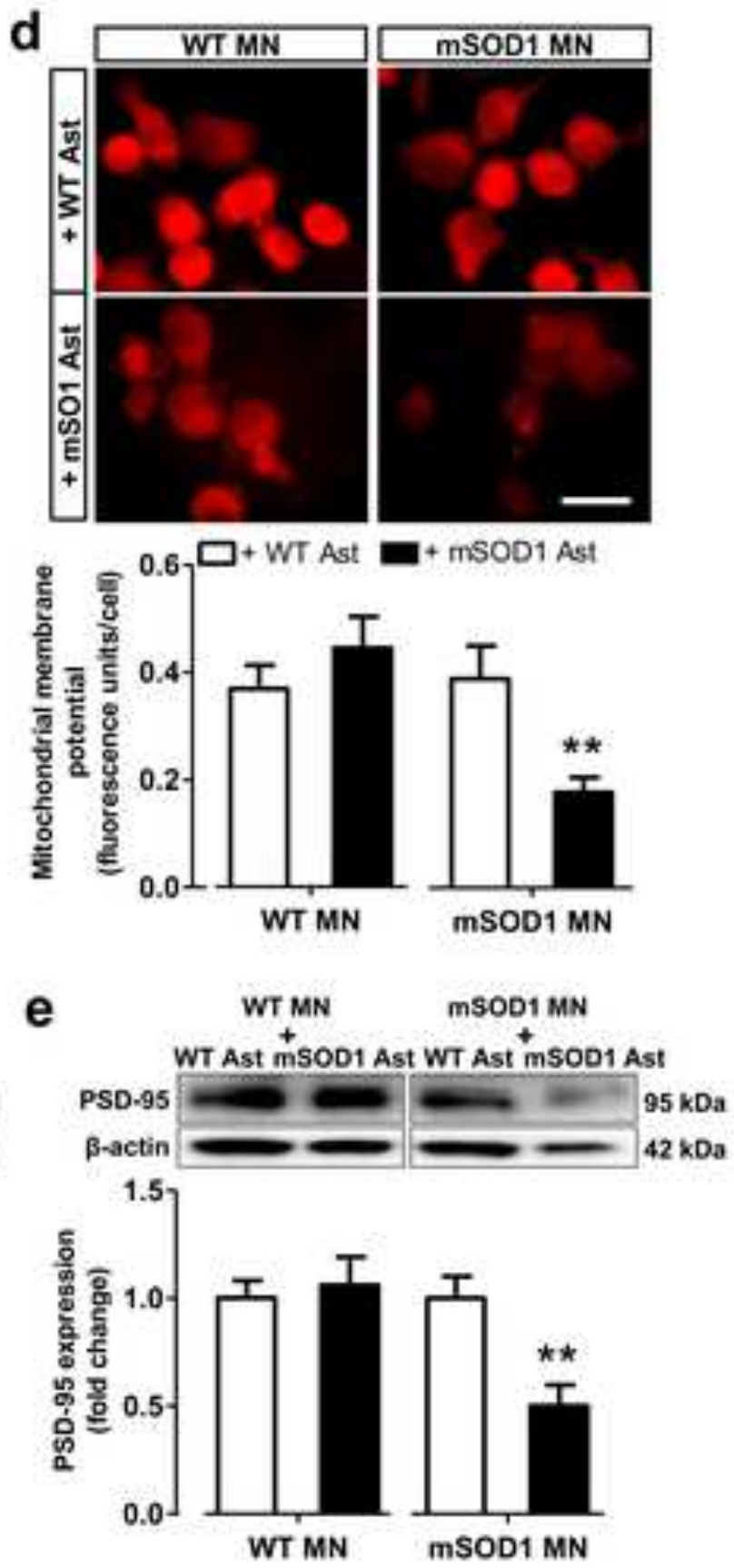

f

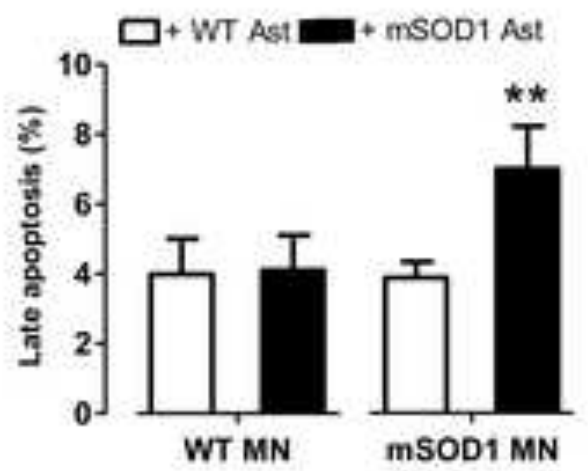

Gomes C. et al. 